\title{
HILGARDIA
}

A Journal of Agricultural Science Published by the California Agricultural Experiment Station

\section{VARIABILITY IN THE MACROFAUNA OF A SINGLE RIFFLE IN PROSSER CREEK, CALIFORNIA, AS INDICATED BY THE SURBER SAMPLER}

Paul R. Needham and Robert L. Usinger 
To determine the variability of the macrofauna in a single riffle, 100 bottom samples were taken in Prosser Creek, Nevada County, California, using the Surber Square Foot Sampler with a Latin Square experimental design. Statistical analyses of the results indicate that $19 \cdot 1$ samples would be required to give significant figures on total wet weights at the 95 per cent level of confidence. Total numbers would require 7.3 samples. The number of square foot samples necessary to ensure representation of the commonest genera or higher groups of aquatics, on the other hand, was quite low; therefore, because of its saving in labor and time, qualitative sampling rather than volume or weight, is recommended for use in pollution studies. Possibly because of the uniformity of the riffle, no correlation was found between types of organisms and different types of bottom, but striking correlations were observed with depth and speed of current. The majority of genera preferred both moderate depths and water speeds although for others the reverse was true. 


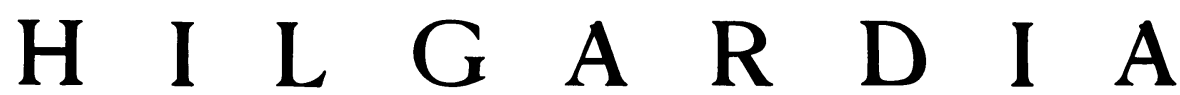

A Journal of Agricultural Science Published by

the California Agricultural Experiment Station

\section{VARIABILITY IN THE MACROFAUNA OF A SINGLE RIFFLE IN PROSSER CREEK, CALIFORNIA, AS INDICATED BY THE SURBER SAMPLER ${ }^{1}$}

\author{
PAUL R. NEEDHAM ${ }^{2}$ and ROBERT L. USINGER ${ }^{3}$
}

\section{INTRODUCTION}

In 1954, the authors designed a plan for a periodic biological sampling program of the macrofauna of streams for the California State Water Pollution Control Board. ${ }^{4}$ In the course of this study and after review of all pertinent literature dealing with the subject of stream-bottom sampling in its qualitative and quantitative aspects, it became obvious that no thorough study had yet been made of the normal variability expected in a single riffle. This held true for both weights and numbers of each major group of organisms present and also for total wet weight of all animals in the individual samples. Accordingly, it was decided that field tests would be conducted to fill this gap in our knowledge of stream ecology. For the purposes of the test it was decided to use the Surber Square Foot Sampler (Surber, 1936) . ${ }^{5}$ This sampler has had the widest use of any of the many types devised.

The Surber sampler is used under a great variety of conditions and for various purposes at the present time. For example, it is used to determine stream productivity as a basis for stocking policies for game fish; it is used to study the foods available to trout and other fishes; and it is used as a means of revealing pollution in streams. However, in none of these cases has it been determined whether or not the method yields results which are statistically significant.

The advantages of the Surber sampler are that it is small, light, easy to carry, and secures samples from a definite area of stream bottom. Its chief disadvantages are: 1) it can be used only in depths up to arms' length ; 2) it can be used only in running water; and 3 ) it permits many organisms to escape in swift water because of back currents at the mouth of the net. In addition, the operator finds it difficult to avoid getting organisms from out-

\footnotetext{
${ }^{1}$ Received for publication June 2, 1955.

2 Professor of Zoölogy-Fisheries, University of California, Berkeley.

${ }^{3}$ Professor of Entomology and Entomologist in the Experiment Station, Berkeley.

- The statistical data from which much of the material presented here was derived are now on file at the office of the State Water Pollution Control Board, Sacramento, California.

${ }^{5}$ See "Literature Cited" for citations, referred to in the text by author and date.
} 
side the square foot frame, since there is no barrier and rocks are almost always disturbed in an effort to set the square frame firmly and evenly on the bottom.

Other types of quantitative samplers are the square foot box sampler (Needham, 1928) the circular square foot sampler (Hess, 1941), dragtype sampler (Usinger and Needham, 1956), and the Ekman (1911) and Peterson (1911) dredges. The latter two types are often used in deep water and soft bottoms. The Peterson is also especially useful for sampling in weed beds.

Still another square foot sampler is the "basket" tray (Wene and Wickliff, 1940). It is a very simple device, but is practical only as a research tool for evaluating the efficiency of other samplers. A square foot of quarterinch mesh screen with wire-stiffened, upturned margins and small side handles is buried in the bottom of a stream in such a way that the normal stream bottom is restored practically to its original state. This is much easier to do in an intermittent stream when the bed is dry, but is possible in a perennial stream. After a period of time the tray is lifted, and with it the square foot of bottom with all associated organisms.

In spite of its limitations, the Surber sampler was chosen for the Prosser Creek test because it has been used so widely in stream survey work.

\section{Description of the Test}

The place selected for the test was a broad and relatively uniform riffle on Prosser Creek near Truckee, California, about one eighth of a mile upstream from the bridge on State Route 89. The riffle was roughly 30 feet wide, and the test area was arbitrarily limited to 100 feet of stream. The bottom was relatively homogeneous, consisting of small gravel and rubble that ranged from particles the size of a pea to stones as large as a grapefruit, intermixed with fine gravel and sand; large rubble was lacking.

The cross-sectional area varied considerably from one side of the stream to the other. On the south side (column 1, fig. 3) the stream bottom "feathered" very gradually to zero depth. On the opposite or north side of the stream where the samples in column 10 were taken, the bank was much steeper, falling away to the deepest and fastest current within a short distance, as shown in figure 1.

Within the sample area 10 vertical columns and 10 horizontal rows were laid out (fig. 1). The resulting checkerboard was then set up as a Latin Square experimental design so that 100 samples could be taken with no one of the five men sampling more than twice in a single column or row. Sampling was started at the downstream row of the riffle in order to avoid disturbance to upstream areas. A stake was driven into the stream bed immediately after taking each sample to mark precisely the point sampled. Two full days were required to take the 100 samples, each man securing 10 samples a day. Ideally, the 100 samples should have been taken simultaneously but this obviously was not possible. The sampling technique is illustrated in figure 2 .

The field test was conducted on July 1 and 2, 1953. Both days were sunny with no overcast. The air temperature ranged from $66^{\circ}$ to $79^{\circ} \mathrm{F}$ during the test. Water temperatures ranged from $48^{\circ} \mathrm{F}$ at 10:00 a.m., July 1 , to $58^{\circ} \mathrm{F}$ at 4:40 p.m., July 2 . The $\mathrm{pH}$ was 7.1 throughout the test. Shade was not a 


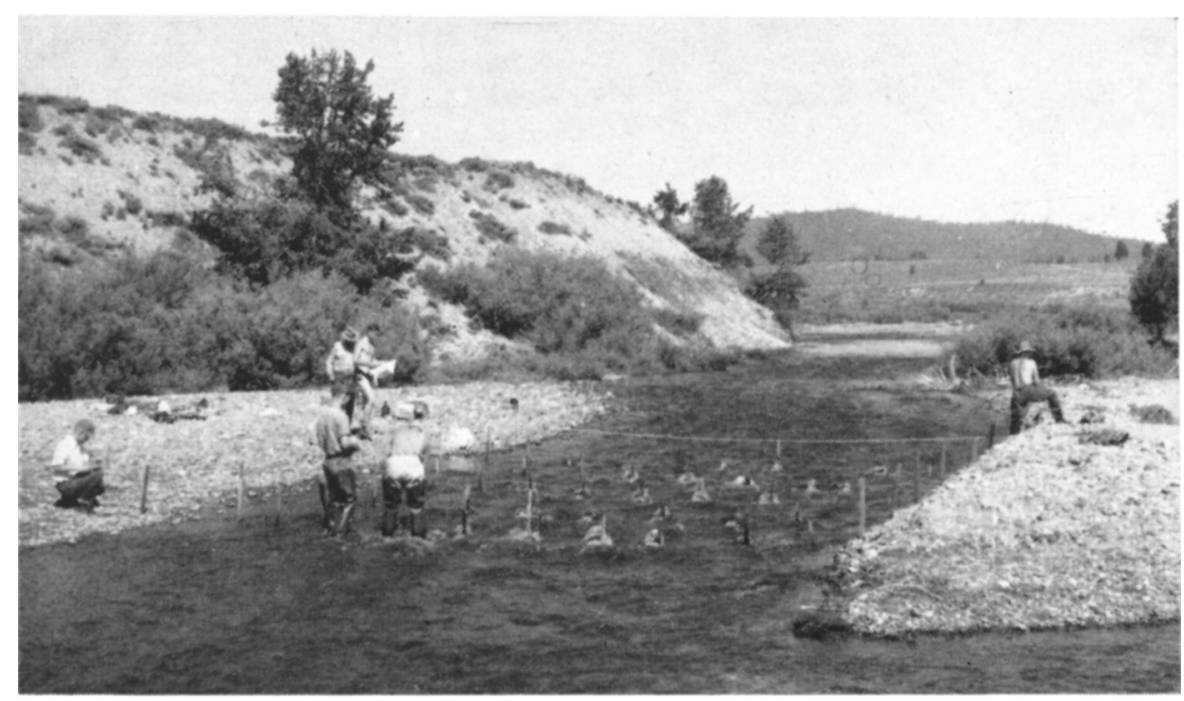

Fig. 1. General view of the test area on Prosser Creek near Truckee, California.

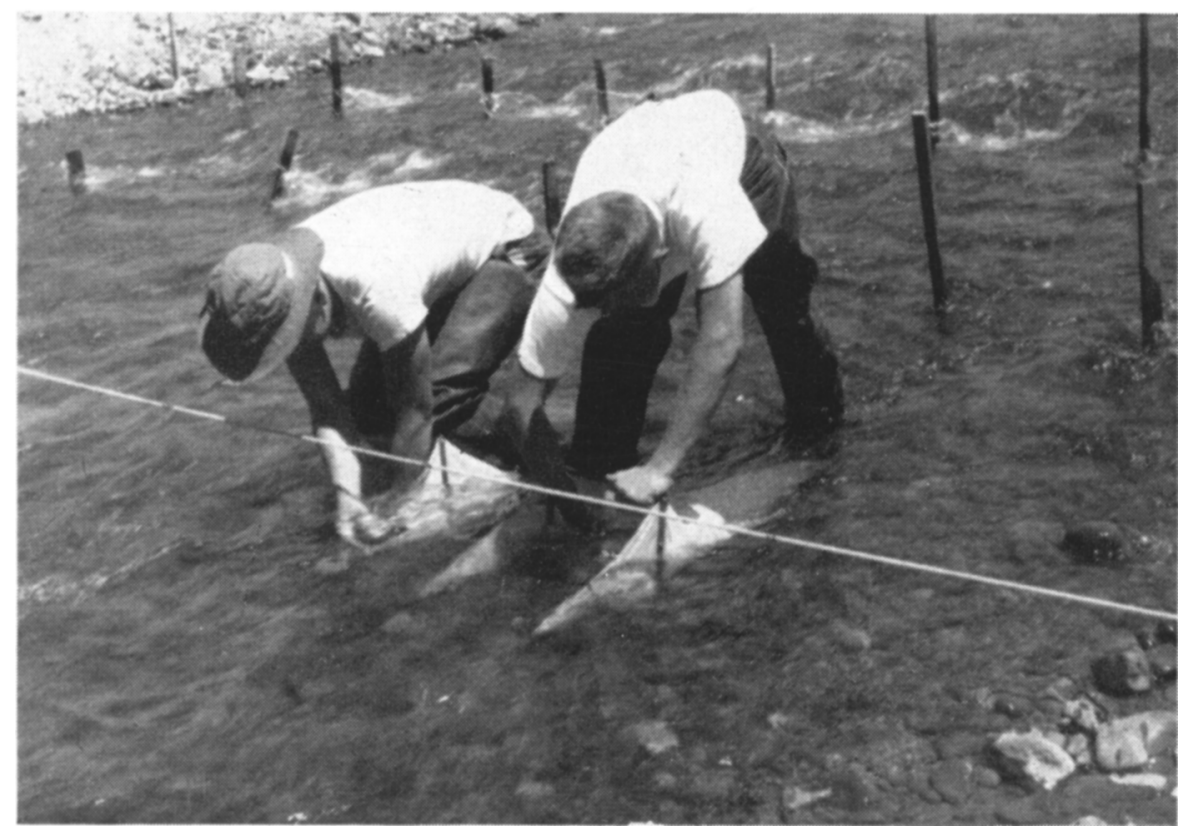

Fig. 2. Sampling technique, using the Surber square foot bottom sampler. 
factor since the riffle was fully exposed to the sun's rays without bank vegetation adjacent to the area sampled.

The 100 preserved samples were picked, weighed, counted, and identified in 3 months immediately following the field test. Picking was done with forceps in a white enamel pan. Wet weights were taken by straining off the alcohol, jarring the specimens onto blotting paper, and weighing after 1 minute of drying. Specimens were then sorted according to taxonomic groups - down to the generic level for insects-and counted as they were placed in vials, 1 vial for each genus.

TABLE 1

COMPARISON OF STREAM WIDTHS MEASURED AT ROWS 1, 5, and 10 AT 10:25 A.M. AND 4:40 P.M. ON JULY 2, 1953

\begin{tabular}{|c|c|c|c|c|c|}
\hline \multirow{2}{*}{ Row number } & \multicolumn{2}{|c|}{ A.M., width in } & \multicolumn{2}{|c|}{ P.M., width in } & \multirow{2}{*}{$\begin{array}{l}\text { Inches } \\
\text { increase }\end{array}$} \\
\hline & Feet & Inches & Feet & Inches & \\
\hline 1. & 29 & 4 & 30 & 2 & 10 \\
\hline 5 .. & 26 & 6 & 27 & 9 & 15 \\
\hline 10. & 24 & 0 & 24 & 9 & 9 \\
\hline
\end{tabular}

\section{Diurnal Fluctuations in Width}

Because of alternate freezing and melting of snow at the head of the Prosser Creek basin, the flow increased slightly in the afternoon of each of the 2 sampling days. This is illustrated by comparing morning and afternoon stream width at rows 1,5 , and 10 (table 1 ). Width increases ranged from 9 to 15 inches at rows 10 and 5 , respectively. The greater increase at row 5 can be attributed to the lower gradient of the south bank at that point in the sampling area.

\section{Depths in the Sampling Area}

Increased afternoon flows, of course, caused parallel diurnal fluctuations in both depth and current speed. Slight increases in depth are more difficult to detect than increments of width because of the great variability in taking depth measurements. Suffice it to say here that on the gentle sloping south bank (column 1), depths at each of the 10 rows sampled ranged from 2.5 inches to 8.0 inches with an average of 3.0 inches (fig. 3 ). Because of insufficient current to wash organisms into the bag of the Surber sampler, it was impossible to sample in shallower water. This would have been undesirable, too, for the additional reason that data obtained by sampling areas intermittently flooded and exposed each day would not be comparable to those from permanently watered, deeper areas. The maximum depth recorded for the 100 samples was 17.0 inches. A profile of the stream bed for each column is presented in figure 3 .

\section{Current Speeds}

Current speeds measured with a Gurley current meter varied from 0.7 of a foot per second at the side of the riffle to 5.27 feet per second in column 6 
near the middle of the riffle at the end of the day when, as pointed out above, peak flows occurred (fig. 3 ).

The rate of flow was also determined by floating a chip down the center of the riffle over the 60 feet of linear distance between rows 1 and 10 and timing it with a stop watch. At 11:10 a.m., on July 2, the rate by the latter method gave a velocity of 5.5 feet per second from the average of 10 tests. At 3:05 p.m. on the same day, a velocity of 5.3 feet per second was determined, or 0.2 feet per second less than that obtained in the morning.

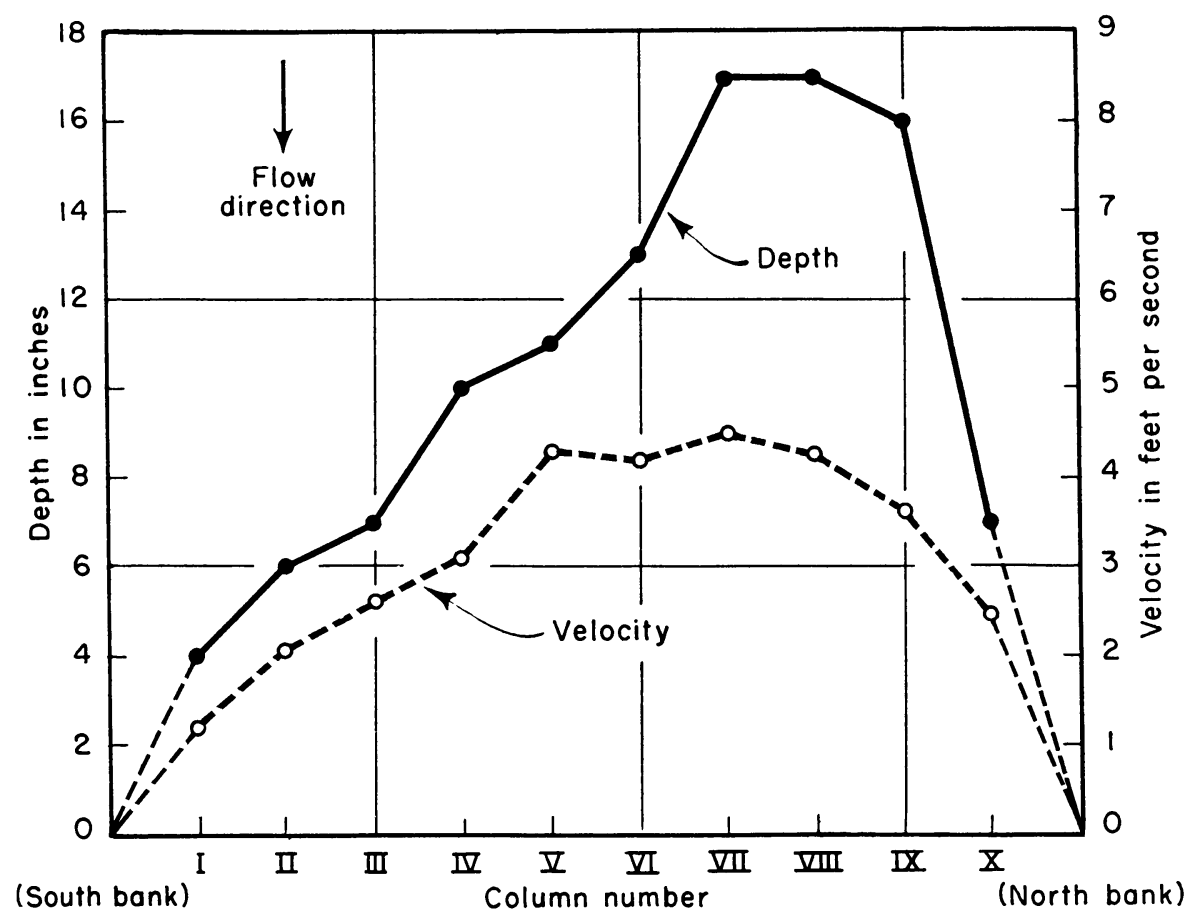

Fig. 3. Water depths and velocities, Prosser Creek, California, recorded across Row 10, July 2, 1953, between $9: 45$ and $10: 45$ a.m.

\section{Questions to be Answered}

As an aid in interpreting previous stream-sampling data and as a guide to future sampling, an attempt was made to find answers to the following basic questions about a single relatively uniform riffle:

1. How many samples are necessary to give statistically significant (95 per cent level) figures on the total number and weight of organisms present?

2. How many samples must be taken to insure getting at least one of each of the groups of organisms present in a single riffle?

3. How much individual variation is there between persons taking the samples?

4. What correlations, if any, exist between types of organisms and the ecological factors of width, depth, speed of current, and type of bottom? 


\section{Results}

Data on wet weights in grams, total numbers of organisms, depths, and persons who took the samples are given in table 2 . The initials of operators stand for Weidlein, Usinger, Jones, Brock, and Helm. Table 2 also shows the Latin Square design with vertical columns and horizontal rows. The direction of flow is from row 1 to row 10. Samples were numbered consecutively from 1 to 100 as taken, beginning with the lower lefthand corner and proceeding across to the lower righthand corner, then back to the left side in row 9 and across to the right, et cetera. (See table 3 for detailed counts according to numbered samples.)

Total Numbers and Weights of Organisms. The statistical analyses revealed that for the whole test, 194 samples would be required to give significant figures on total weights of organisms, and 73 samples would be necessary to give significant figures on total numbers at a 95 per cent level of significance. The greater variability of weights of the individual samples compared with numbers is further indicated by the coefficients of variation. These were 0.78 for weights and 0.56 for numbers. Obviously no fishery biologist, ecologist, or other investigator would have either the time or the energy to take 194 or 73 samples from any given riffle. We then can conclude from these data that purely quantitative routine sampling in streams to determine weights and numerical data is impractical. Wet weights ranged in variation from a low of 0.015 gram (sample no. 8) to a high of 2.31 grams (sample no. 95) with an overall average of 0.575 gram. Total numbers in each sample varied from 2 (sample no. 8) to 198 (sample no. 44). The average number was 75.7 .

Number of Samples Required to Insure Representation of Each Group of Organisms. While total weights of organisms showed tremendous variation, the frequency of occurrence of the abundant or common kinds or groups of organisms was much less variable. For this reason and because weighing of a sample is far more laborious than counting, only the numbers of the common genera were used in the course of the detailed analysis of the Prosser Creek results. Total counts for each genus (or higher group) in each sample are given in table 3. For purposes of this analysis, groups of organisms which were represented by very low densities were excluded. A list of the common groups is given in table 4, together with the approximate number of samples required to insure getting at least 1 representative of each group.

The groups for which small numbers of samples are required, all belonging to the Plecoptera, Ephemeroptera, Trichoptera, and Diptera, were represented by large numbers of organisms and were therefore appropriate for statistical analysis. Of these, only the Plecoptera genera Isogenus, Isoperla, and Alloperla followed a Poisson distribution. The lack of fit of the other groups merely requires that the binomial distribution be applied. In table 4 a collective figure is given for the whole order after the genera contained in the order.

Sample calculations are given below for both Poisson (Alloperla) and binomial (Brachycentrus) distributions. In both cases it is assumed that there was complete independence of samples. 
TABLE 2

LATIN SQUARE DESIGN OF THE PROSSER CREEK TEST*

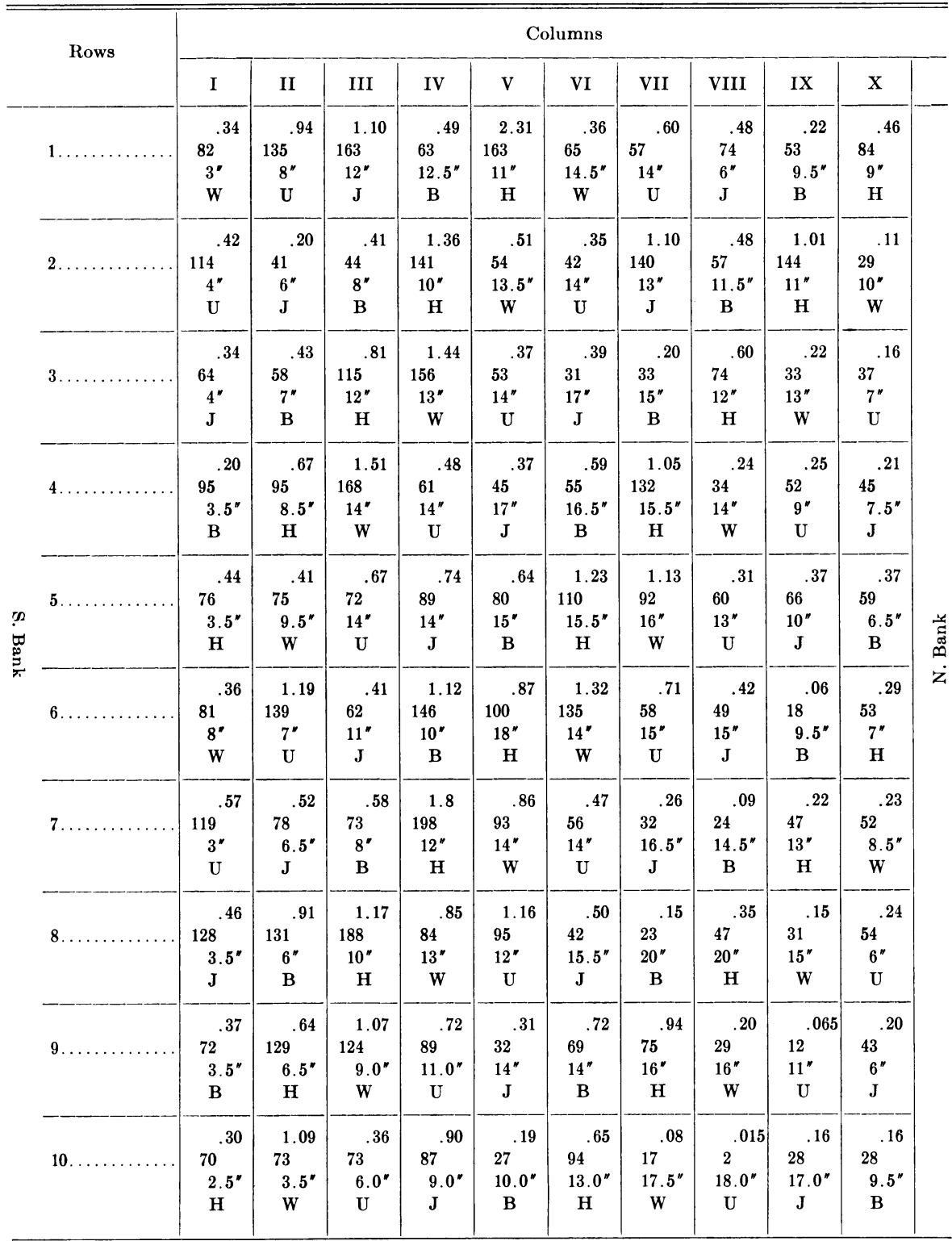

Direction of Flow $\downarrow$

- In each square from top to bottom is given, in order, the wet weight in grams of organisms, the number of organisms, depth, and initial of the person who took the sample. 
TABLE 3

ORIGINAL DATA ARRANGED ACCORDING TO SEQUENCES OF SAMPLES IN THE LATIN SQUARE EXPERIMENTAL DESIGN

\begin{tabular}{|c|c|c|c|c|c|c|c|c|c|c|c|c|c|c|c|c|c|c|c|c|c|c|}
\hline \multirow{2}{*}{$\begin{array}{c}\text { (ienera } \\
\text { (or higher groups) }\end{array}$} & \multirow[b]{2}{*}{1} & \multirow[b]{2}{*}{2} & \multirow[b]{2}{*}{3} & \multirow[b]{2}{*}{4} & \multirow[b]{2}{*}{5} & \multirow[b]{2}{*}{6} & \multirow[b]{2}{*}{7} & \multirow[b]{2}{*}{8} & \multirow[b]{2}{*}{9} & \multirow[b]{2}{*}{10} & \multirow[b]{2}{*}{11} & \multirow[b]{2}{*}{12} & \multirow[b]{2}{*}{13} & & & & & & & & & \\
\hline & & & & & & & & & & & & & & 14 & 15 & 16 & 17 & 18 & 19 & 20 & 21 & 22 \\
\hline Nematoc & .. & .. & . & . & 1 & .. & .. & .. & .. & $\cdots$ & . & $\cdots$ & .. & $\ldots$ & $\ldots$ & $\cdots$ & .. & 1 & .. & .. & .. & \\
\hline ligochaetes.. & 2 & .. & .. & . & .. & .. & .. & . & . & .. & .. & . & .. & .. & .. & . & .. & .. & . & .. & .. & 1 \\
\hline Hydracarina.... & .. & 1 & .. & . & .. & .. & . & .. & & . & .. & . & .. & .. & . & . & .. & . & .. & . & . & .. \\
\hline Alloperla. . & 4 & 4 & 1 & . & 1 & 3 & . & . & 1 & 1 & 2 & 1 & 2 & 2 & . & 2 & 2 & . & . & 4 & 8 & \\
\hline Isogenus. . & 1 & . & . & . & . & . & . & . & . & .. & .. & 2 & . & . & . & .. & 1 & . & .. & . & .. & \\
\hline Nemoura.. & . & . & . & . & & & . & . & & .. & . & .. & . & .. & . & .. & . & .. & . & $\ldots$ & 1 & .. \\
\hline Isoperla......... & 1 & . & . & 1 & . & 1 & . & . & . & .. & 1 & .. & . & .. & .. & . & 2 & . & .. & 1 & 3 & .. \\
\hline Arcynopteryx... & .. & . & .. & . & . & .. & . & .. & . & . & . & .. & . & $\cdots$ & .. & .. & .. & .. & $\cdots$ & . & . & .. \\
\hline Buetis. & . & 2 & 2 & 3 & 1 & 6 & 2 & .. & 2 & 2 & $\cdots$ & 9 & 3 & 5 & 2 & 3 & 2 & .. & .. & 4 & 6 & 5 \\
\hline Amelet & 3 & & 1 & . & . & . & . & . & . & & 2 & . & . & .. & .. & . & .. & . & .. & .. & 4 & .. \\
\hline Cinyg & 25 & 15 & 20 & 13 & 4 & 5 & . & .. & 1 & 11 & 19 & 37 & 20 & 13 & 3 & 1 & 1 & 1 & 3 & 11 & 25 & 18 \\
\hline Rhith & .. & 3 & 5 & 5 & 2 & 36 & 1 & . & 2 & . & 1 & 6 & 3 & 10 & 3 & 14 & 27 & 4 & 3 & 6 & 1 & 1 \\
\hline Iron. . & . & 6 & 9 & 11 & 3 & 7 & 6 & . & 15 & 1 & 6 & 4 & 6 & 6 & 3 & 1 & 9 & . & 1 & 3 & 16 & 6 \\
\hline Ephemerella. & 16 & 17 & 16 & 8 & 3 & 11 & 1 & 1 & 1 & 5 & 19 & 38 & 25 & 9 & 4 & 5 & 9 & 4 & 1 & 8 & 13 & 23 \\
\hline lebia. & . & . & . & . & .. & .. & .. & .. & .. & .. & .. & .. & .. & . & . & .. & . & . & .. & . & .. & .. \\
\hline & 1 & .. & .. & .. & .. & .. & 1 & 1 & .. & .. & .. & .. & $\ldots$ & 1 & .. & $\ldots$ & .. & 1 & .. & & 1 & \\
\hline Gilo & . & 3 & 9 & 44 & 9 & 13 & .. & .. & . & .. & 2 & 7 & 28 & 22 & 7 & 33 & 12 & 14 & 1 & .. & .. & 53 \\
\hline he... & 2 & 3 & 3 & . & . & .. & .. & .. & . & .. & .. & 7 & 2 & .. & .. & .. & .. & .. & .. & 1 & 3 & .. \\
\hline Brachycen & 1 & 2 & 4 & . & 1 & 1 & 2 & .. & 1 & $\ldots$ & .. & 4 & 9 & .. & .. & $\ldots$ & 1 & . & . & 1 & .. & 2 \\
\hline Lepidosto & 12 & 2 & . & 1 & . & 2 & . & . & . & 2 & 8 & 4 & 10 & 2 & 1 & 1 & 2 & . & . & 1 & 17 & 3 \\
\hline hila.... & .. & . & . & . & .. & .. & . &.. & . & .. & .. & .. & 2 & 1 & 2 & .. & . & . & .. & . & 2 & 2 \\
\hline$\ldots \ldots$ & .. & . & .. & . & .. & .. & .. & .. & .. & .. & .. & .. & 1 & .. & .. & .. & .. & .. & . & . & .. & .. \\
\hline Limn & .. & 1 & .. & . & .. & .. & .. & .. & . & .. & .. & .. & .. & .. & .. &.. & .. & .. & .. & .. & 1 & .. \\
\hline ilidae... & . & .. & . & . & .. & . & .. & .. & . & . & . & .. & .. & .. & . & $\ldots$ & $\ldots$ & . & . & .. & .. & .. \\
\hline dae..... & . & .. & $\ldots$ & $\ldots$ & $\ldots$ & $\ldots$ & .. & .. & . & . & .. & .. & .. & .. & $\ldots$ & .. & $\ldots$ & . & . & .. & .. & .. \\
\hline$\ldots \ldots$ & $\ldots$ & $\ldots$ & $\ldots$ & . & . & $\ldots$ & $\ldots$ & .. & $\ldots$ & . & $\ldots$ & .. & .. & $\ldots$ & .. & .. & $\ldots$ & $\ldots$ & . & . & .. & .. \\
\hline Elm & $\ldots$ & .. & . & . & $\ldots$ & . & $\ldots$ & .. & . & . & $\ldots$ & .. & 1 & 1 & .. & & 1 & . & $\ldots$ & .. & 1 & 2 \\
\hline Psepher & . & .. & . & . & .. & . & .. & .. & . & . &.. & .. & $\ldots$ & .. & .. & .. & .. &.. &.. & .. & . & .. \\
\hline S S & & 1 & 1 & 1 & 1 & 5 & 3 & . & 1 & 2 & $\cdots$ & 1 & 2 & 3 & & .. & 3 & 2 & 1 & 1 & 1 & 2 \\
\hline & . & . & .. & . & $\ldots$ & $\ldots$ & .. & . & . & . & . & .. & $\ldots$ & . & $\ldots$ & .. & .. & $\ldots$ & . & $\ldots$ & 3 & \\
\hline Chir & . & 6 & 1 & . & .. & .. & .. & .. & 2 & .. & 3 & 3 & 5 & 8 & 4 & 6 & 2 & 2 & 1 & 1 & 10 & 9 \\
\hline & .. & .. & .. & .. & .. & .. & . & .. & . & .. & .. & .. & .. & .. & .. & .. & .. & . & .. & .. & 2 & .. \\
\hline Eri & . & .. & . & . & .. & .. & . & .. & $\therefore$ & .. & .. & .. & 1 & .. & .. & . & .. & .. & $\cdots$ & & .. & .. \\
\hline Hexatoma....... & 2 & 4 & . & . & . & .. & .. & .. & . & .. & 7 & 1 & 1 & .. & 1 & 1 & . & . & . & . & 10 & 1 \\
\hline Eriocera.. & . & 2 & . & . & . & .. & . & .. & . & .. & 1 & .. & 3 & 2 & .. & .. & .. & . & .. & . & .. & .. \\
\hline Blepharoceridae. & . & . & . & . & . & . & . & . & . & . & . & . & . & . & . & . & . & . & . & . & . & .. \\
\hline Antocha......... & . & 1 & . & . & . & $\ldots$ & $\cdots$ & . & . & . & . & . & . & 1 & .. & 1 & 1 & . & . & . & . & .. \\
\hline Tak & . & .. & . & . & . & . & . & . & . & . & . & . & . & .. & . & .. & .. & . & . & . & .. & . \\
\hline Tipulidae & . & . & . & . & . & . & . & .. & . & . & . & . & . & . & . & .. & . & .. & . & . & .. & .. \\
\hline Nos & & & & & & & & & & & & & & & & & & & & & & \\
\hline & . & . & .. & .. & $\cdots$ & . & $\cdots$ & . & . & .. & $\cdots$ & 4 & . & . & . & . & .. & . & . & . & . & .. \\
\hline & .. & . & $\therefore$ & & . & . & . & . & . & .. & . & .. & .. & .. & .. & .. & . & . & . & . & . & .. \\
\hline & .. & . & . & .. & .. & .. & .. & .. & . & .. & .. & .. & .. & .. & .. & .. & .. & $\cdots$ & .. & . & .. & 1 \\
\hline & .. & . & . & . & .. & .. & .. & .. & .. & .. & .. & .. & .. & .. & .. & .. & .. & .. & .. & . & .. & .. \\
\hline Cyclorrhapha.. & . & . & . & . & .. & . & . & . & . & . & . & .. & . & .. & .. & .. & . & .. & . & . & .. & .. \\
\hline
\end{tabular}




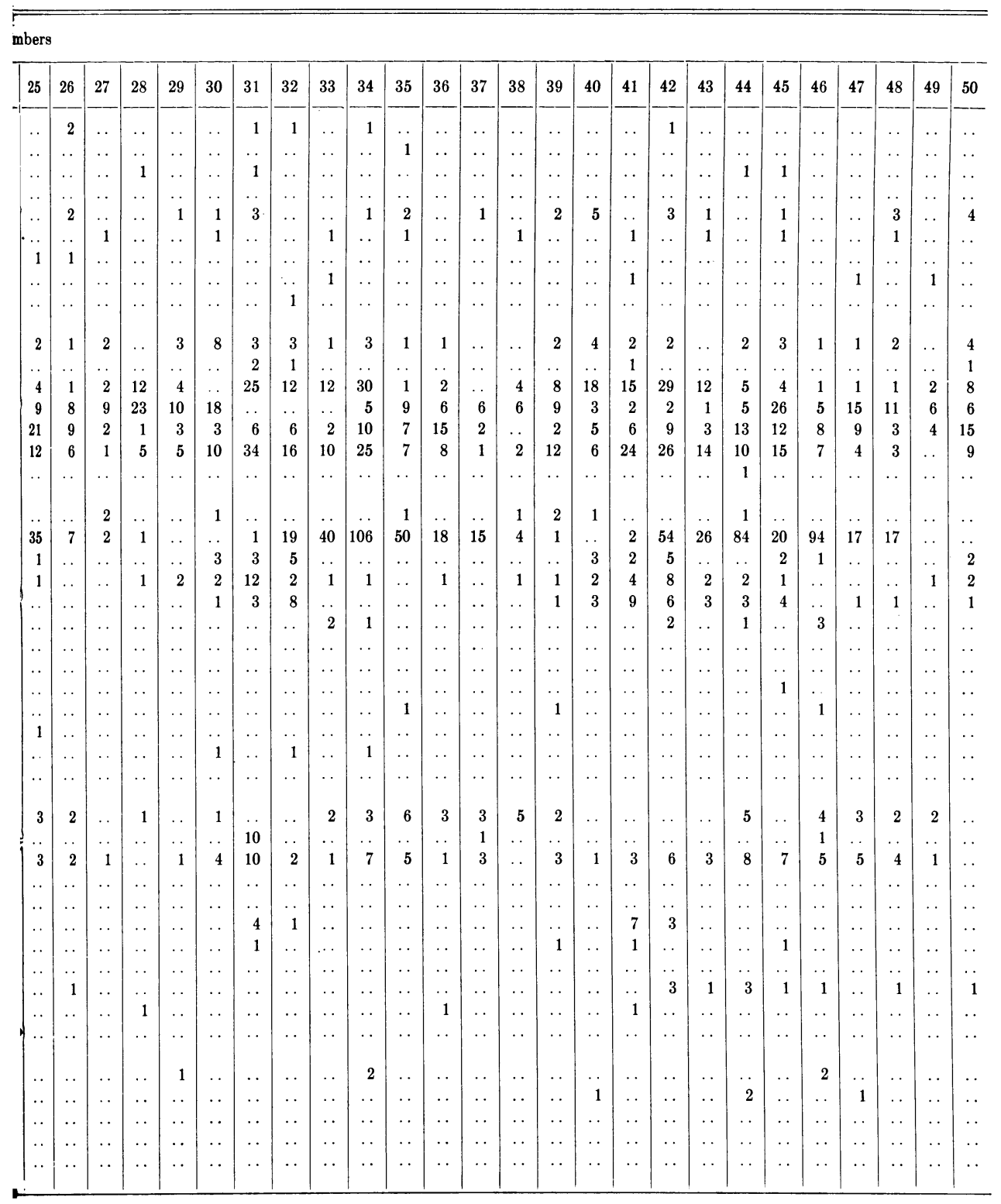


TABLE 3

(Concluded)

\begin{tabular}{|c|c|c|c|c|c|c|c|c|c|c|c|c|c|c|c|c|c|c|c|c|c|c|}
\hline \multirow{2}{*}{$\begin{array}{c}\text { Genera } \\
\text { or higher groups) }\end{array}$} & \multicolumn{22}{|c|}{ Sample } \\
\hline & 51 & 52 & 53 & 54 & 55 & 56 & 57 & 58 & 59 & 60 & 61 & 62 & 63 & 64 & 65 & 66 & 67 & 68 & 69 & 70 & 71 & 72 \\
\hline Nematodes... & . & . & . & . & . & . & .. & . & . & .. & 1 & . & .. & . & . & . & .. & . & $\cdots$ & . & . & .. \\
\hline Oligochaetes..... & . & .. & . & . & .. & .. & .. & .. & 1 & .. & . & .. & .. & .. & . & .. & .. & . & . & . & $\cdots$ &.. \\
\hline Hydracarina.... & .. & .. & .. & .. & .. & .. & .. & .. & .. & .. & .. & .. & .. & .. & .. & .. & .. & .. & .. & .. & 1 &.. \\
\hline Alloperla.. & 3 & 1 & . & 1 & 3 & 3 & 2 & 1 & 2 & 2 & 5 & . & 4 & 1 & 2 & 1 & 3 & 1 & 1 & 2 & 6 & 1 \\
\hline Isogenus . . . . . . . & . & .. & .. & .. & .. & .. & .. & 1 & 1 & .. & .. & 1 & .. & 1 & .. & .. & 1 & .. & .. & 1 &.. & .. \\
\hline Nemoura......... & .. & .. & .. & .. &. & .. & .. & . & . & . & . & . & .. & .. & .. & .. & . & .. &. &. & .. &.. \\
\hline Isoperla.......... & .. & .. & .. & .. & .. & .. & .. & 1 & . & .. & 2 & 1 & .. & .. & . & .. & .. & .. & 2 & 2 & .. &.. \\
\hline Arcynopteryx.... & .. & .. & .. & .. & .. & . & .. & .. & 1 & .. & .. & .. & .. & . & $\therefore$ & .. & . & .. & .. & .. & . &.$\cdot$ \\
\hline Baetis...... & .. & . & 1 & 1 & 2 & 2 & 1 & 3 & 6 & 2 & .. & 2 & 6 & 3 & .. & .. & 4 & 2 & 3 & 1 & .. & 1 \\
\hline Ameletus.... & 17 & .. & . & .. & .. & 1 & . & . & .. & . & 4 & .. & .. & . & . & . &. & . & . & . & .. &.. \\
\hline Cinygmula....... & 8 & 17 & 3 & 3 & 5 & 5 & 2 & 4 & 6 & 9 & .. & 11 & 10 & 3 & 1 & 1 & 16 & 4 & 9 & 9 & 10 & 12 \\
\hline Rhithrogena..... & . & .. & 1 & 2 & 9 & 17 & 12 & $\mathbf{5}$ & 12 & 4 & .. & .. & 7 & 9 & 7 & 6 & 25 & 5 & 17 & 7 & .. & 3 \\
\hline Iron............ & 1 & 4 & . & 2 & 5 & 18 & 3 & .. & 3 & 16 & 2 & 2 & 19 & 6 & 1 & 1 & 19 & 3 & 4 & 6 & 3 & .. \\
\hline Ephemerella..... & 3 & 10 & 9 & 3 & 12 & 8 & 5 & 11 & 17 & 7 & 9 & 33 & 21 & 5 & 2 & .. & 9 & 7 & 4 & 7 & 10 & 8 \\
\hline Paraleptophlebia. & 1 & . & .. & .. & .. & .. & .. & .. & . & . & 1 & .. & .. & .. & .. & $\ldots$ & .. &.. & .. & .. & .. & 1 \\
\hline Sericostoma.. & 2 & .. & .. & 2 & .. & 1 & . & 2 & . & 1 & 3 & . & .. & . & . & 1 & .. & 1 & . & .. & 1 & .. \\
\hline Glossosoma.... & . & 21 & 47 & 56 & 28 & 42 & 54 & 3 & 3 & . & 1 & 33 & 74 & 17 & 30 & 39 & 37 & 7 & 1 & .. & 1 & 19 \\
\hline Hydropsyche... & . & 1 & .. & . & 1 & .. & .. & .. & 1 & . & . & 2 & 1 & .. & .. & 1 & 1 & .. & .. & 3 & 1 & .. \\
\hline Brachycentrus.. & .. & 3 & . & 1 & 2 & .. & .. & 4 & . & 1 & . & 1 & . & 2 & .. & .. & 1 & .. & 1 & 1 & 2 & 6 \\
\hline Lepidostoma.... & 12 & 1 & 2 & 3 & 1 & 1 & .. & 10 & .. & 2 & 9 & 1 & 4 & .. & .. & .. & 1 & .. & 1 & 2 & 8 & 3 \\
\hline Rhyacophila... & .. & .. & 1 & .. & 1 & 1 & .. & 1 & .. & .. & .. & . & 2 & 1 & .. & .. & 1 & .. & .. & .. & 1 & .. \\
\hline Neophylax.... & . & .. & .. & .. & . & .. & .. & .. & .. & .. & .. & .. & . & .. & .. & .. & .. & .. & .. & .. & .. & .. \\
\hline Limnephilus.... & 2 & . & .. & .. & .. & .. & .. & .. & .. & .. & . & . & . & .. & . & . & .. & .. & .. & .. & .. & .. \\
\hline Limnephilidae... & .. & 1 & .. & .. & . & .. & .. & .. & .. & .. & 1 & . & 1 . & . & .. & . & .. & .. & .. & . & .. & .. \\
\hline Dytiscidae..... & .. & .. & . & .. & 1 & . & .. & . & .. & . & 2 & . & .. & 1 & .. & .. & . & .. & .. & .. & .. & .. \\
\hline Helophorus...... & .. & .. & .. & .. & . & . & .. & .. & .. & .. & .. & 1 & .. & . & . & .. & .. & . & .. & .. & .. & .. \\
\hline Elmus . . . . . . . . & .. & .. & .. & .. & . & 1 & .. & 1 & . & . & .. & . & .. & . & .. & .. & . & .. & .. & .. & .. & .. \\
\hline Psephenidae..... &.. & .. & . & $\cdots$ & . & .. & . & . & .. & .. & $\cdots$ & . & .. & . & .. & . & . & .. & .. & .. & .. & .. \\
\hline Simuliidae. . & . & 1 & 1 & 3 & 2 & 4 & 3 & 8 & 7 & 5 & 1 & .. & 5 & 3 & .. & 2 & 5 & .. & 6 & 1 & .. & 1 \\
\hline Heleidae........ & 2 & 1 & .. & .. & . & .. & . & .. & . & 2 & 8 & . & . & . & . & .. & & . & . & .. & .. & .. \\
\hline Chironomidae.... & 17 & 10 & $\mathbf{5}$ & 12 & 1 & 5 & 9 & 2 & 6 & 6 & 21 & 5 & 7 & 2 & 3 & 3 & 8 & 3 & 3 & 3 & 13 & 1 \\
\hline Limnophila...... & .. & . & .. & .. & .. & .. & .. & .. & . & .. & .. & . & .. & . & .. & .. & .. & .. & .. & .. & .. & .. \\
\hline Erioptera...... & . & . & .. & .. & .. & .. & .. & . & .. & .. & .. & . & .. & .. & . & .. & . & . & .. & . & . & .. \\
\hline Hexatoma....... & 5 & 4 & 1 & .. & .. & .. & . & . & . & .. & 15 & 1 & 2 & .. & . & .. & .. & .. & .. & .. & 4 & 1 \\
\hline Eriocera.......... & 2 & .. & $\cdots$ & . & .. & .. & 1 & .. & . & .. & .. & . & 1 & .. & .. & .. & . & .. & .. & .. & .. & .. \\
\hline Blepharoceridae. . & .. & . & .. & .. & .. & . & .. & .. & .. & .. & . & . & 1 & . & .. & .. & 1 & .. & .. & .. & .. & .. \\
\hline Antocha......... & .. & . & . & . & 1 & .. & .. & .. & .. & .. & .. & 1 & .. & 1 & . & .. & .. & .. & . & . & .. & .. \\
\hline Tabanidae...... & .. & .. & .. & .. & .. & .. & .. & . & .. & .. & 1 & .. & .. & .. & .. & .. & .. & .. & .. & .. & .. & .. \\
\hline Tipulidae..... & .. & .. & .. & .. & .. & .. & .. & .. & .. & .. & 1 & .. & . & .. & .. & . & .. & .. & .. & .. & .. & .. \\
\hline $\begin{array}{l}\text { Nostoc Chiro- } \\
\text { nomidae...... }\end{array}$ & . . & $\ldots$ & $\ldots$ & $\ldots$ & 5 & $\ldots$ & $\ldots$ & 1 & $\ldots$ & $\ldots$ & 6 & $\ldots$ & $\ldots$ & 6 & $\ldots$ & $\ldots$ & $\ldots$ & 1 & $\ldots$ & $\ldots$ & .. & 1 \\
\hline Pericoma......... & . & .. & 1 & .. & .. & .. & .. & .. & . & .. & 1 & . & .. & . & .. & .. & .. & .. & .. & .. & .. & .. \\
\hline Dolichopodidae. . & .. & .. & .. & .. & .. & .. & .. & .. & . & .. & .. & . & .. & .. & . & .. & .. & .. & .. & .. & .. & .. \\
\hline Rhagionidae.... & .. & . & .. & . & . & .. & .. & 1 & . & .. & . & . & 1 & .. & .. & . & .. & .. & .. & .. & .. & .. \\
\hline Cyclorrhapha.... & $\ldots$ & .. & & .. & & .. & $\cdots$ & $\ldots$ & .. & .. & .. &.. & $\cdots$ & .. &.. & .. & .. &.. & .. & .. &.. & .. \\
\hline
\end{tabular}




\begin{tabular}{|c|c|c|c|c|c|c|c|c|c|c|c|c|c|c|c|c|c|c|c|c|c|c|c|c|c|c|}
\hline \multicolumn{27}{|c|}{ umbers } \\
\hline 74 & 75 & 76 & 77 & 78 & 79 & 80 & 81 & 82 & 83 & 84 & 85 & 86 & 87 & 88 & 89 & 90 & 91 & 92 & 93 & 94 & 95 & 96 & 97 & 98 & 99 & 100 \\
\hline 1 & .. & .. & .. & $\ldots$ & $\ldots$ & & $\ldots$ & $\ldots$ &.. & $\ldots$ & $\ldots$ & $\ldots$ & $\ldots$ & $\ldots$ & . & $\ldots$ & $\ldots$ & $\ldots$ & $\ldots$ & $\cdots$ & . & $\ldots$ & $\cdots$ & .. & $\ldots$ & $\ldots$ \\
\hline .. & .. & .. & .. & $\ldots$ &.. & .. & $\ldots$ & .. &.. & .. & .. & .. & .. & $\ldots$ & .. & .. & . & .. &.. & .. &.. & $\ldots$ &.. & 1 &.. & . \\
\hline .. &.. &.. & .. & 1 &.. & .. &.. & .. &.. & .. & .. &.. & .. &.. &.. & . & .. & .. &.. & .. &. & .. & .. & .. & .. & .. \\
\hline 4 & 1 & .. & 2 & 3 & 2 & 5 & 6 & 4 & .. & .. & 2 & 1 & .. & .. & . & 1 & 4 & 3 & 3 & 1 & . & 1 & . & . & 3 & 4 \\
\hline .. & . & .. & .. & 2 & .. & .. & 3 & 1 & 1 & . & .. & .. & .. & .. & 1 & .. & $\ldots$ & 2 & .. & . & .. & $\ldots$ & .. & .. & .. & 2 \\
\hline.. &.. & .. & .. & 1 & . & . & 1 & . & .. & .. & . & .. & . & .. & .. & .. & . & . & . & .. &. & .. & .. & . & .. & . \\
\hline .. & .. & .. & .. & .. & . & .. & .. & .. & .. & .. & 1 & .. & . & . & .. & . & 2 & 1 & . & .. & 1 & . & . & 1 & .. & . \\
\hline .. &.. & .. & .. & $\ldots$ & $\because$ & .. &.. & .. & $\ldots$ & .. & .. & .. & .. &.. & .. & .. & .. & . & $\ldots$ & .. &.. & $\ldots$ & .. &.. &. & . \\
\hline 7 & 5 & 2 & 1 & 12 & 1 & 2 & 3 & 1 & 1 & 2 & 4 & 1 & 4 & 1 & 4 & . & 1 & 2 & $\ldots$ & 2 & 4 & 3 & 1 & 4 & $\ldots$ & 4 \\
\hline 1 & $\ldots$ & $\ldots$ & $\ldots$ & $\ldots$ & $\ldots$ & 1 & 1 & $\ldots$ & $\ldots$ & $\ldots$ & $\ldots$ & $\ldots$ & $\ldots$ & $\ldots$ & $\ldots$ & $\ldots$ & $\ldots$ & $\ldots$ & $\ldots$ & $\ldots$ & $\ldots$ & $\ldots$ & & $\ldots$ & $\ldots$ & $\ldots$ \\
\hline 8 & 5 & $\ldots$ & 2 & 12 & 3 & 7 & 28 & 10 & 6 & 7 & 3 & 1 & 2 & 4 & 9 & 3 & 25 & 22 & 13 & $\ldots$ & 10 & $\ldots$ & 2 & 1 & 9 & 24 \\
\hline$B$ & 10 & 6 & 5 & 10 & 7 & 5 & 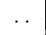 & 1 & 6 & 5 & 11 & 14 & 5 & 20 & 5 & 7 & $\ldots$ & 4 & 4 & 6 & 34 & 14 & 5 & 10 & 17 & 11 \\
\hline 11 & 4 & .. & 5 & 7 & 3 & 7 & 9 & $\ldots$ & 6 & 29 & 4 & 4 & 33 & 3 & 7 & 3 & 8 & 21 & 26 & 7 & 33 & 10 & 13 & 8 & 3 & 8 \\
\hline II & 7 & 1 & $\ldots$ & 3 & 3 & 4 & 20 & 7 & 6 & 15 & 7 & 6 & 16 & 3 & 20 & 6 & 7 & 15 & 27 & 5 & 10 & 5 & 7 & 8 & 14 & 14 \\
\hline .. &.. & .. &.. & 1 & .. & $\ldots$ &.. & 1 & $\ldots$ & $\ldots$ &.. & .. & $\ldots$ & .. & $\ldots$ & .. & 2 & $\ldots$ &.. & .. & $\ldots$ & .. &.. & $\ldots$ & $\ldots$ &.. \\
\hline 1 & . & .. & . & 2 & 1 & $\ldots$ & 2 & $\ldots$ & $\ldots$ & .. & 2 & 1 & $\ldots$ & 1 & 1 & $\ldots$ & .. & 1 & 1 & 2 & 2 & $\ldots$ & 1 &.. & . & 1 \\
\hline 7 & 9 & 16 & 7 & 10 & 5 & 1 & 2 & 8 & 13 & 59 & 15 & 4 & 59 & 18 & 8 & $\ldots$ & 5 & 45 & 22 & 27 & 55 & 7 & 18 & 14 & 1 & .. \\
\hline 1 & .. & .. & .. & 4 & 1 & . . & 1 & .. & 1 & 1 & $\ldots$ & .. & 1 & $\ldots$ & . & $\ldots$ & . & 2 & 2 & 2 & $\ldots$ & . & $\ldots$ & 1 & 1 & 1 \\
\hline 7 & .. & .. & & $\cdots$ & .. & - & 1 & 3 & . & 2 & .. & . & 1 & & 3 & $\ldots$ & 7 & 3 & 4 & 2 & 3 & 1 & . & 10 &. & 6 \\
\hline 7 & 1 & 1 & 1 & 2 & $\ldots$ & 1 & 8 & 3 & 3 & 3 & 1 & 2 & 4 & 2 & 6 & $\ldots$ & 10 & 7 & 2 & $\ldots$ & 3 & $\ldots$ & $\ldots$ & $\ldots$ & 1 & 3 \\
\hline . & 1 & 1 & $\ldots$ & $\ldots$ & $\ldots$ & $\ldots$ & $\ldots$ & .. & $\ldots$ & 1 & 1 & $\ldots$ & 2 & $\ldots$ & 2 & $\ldots$ & $\ldots$ & 1 & 2 & 1 & $\ldots$ & $\ldots$ & . & 1 & $\ldots$ & 2 \\
\hline$\cdot$ & 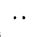 &.. &.. &.. & . & $\ldots$ &.. & .. & $\ldots$ & . & $\ldots$ & $\ldots$ & . & $\ldots$ & $\ldots$ & $\ldots$ & $\ldots$ & $\ldots$ & $\ldots$ & $\ldots$ & $\ldots$ & .. & $\ldots$ & . & $\ldots$ & .. \\
\hline .' & $\cdots$ &.. & .. &.. & $\ldots$ & .. & .. & .. &.. & .. & $\ldots$ &.. & . &.. & .. &.. &.. & .. & .. &.. & $\ldots$ & . & $\ldots$ & .. & $\ldots$ &.. \\
\hline .. & 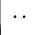 &.. &.. & $\ldots$ & $\ldots$ & $\ldots$ & $\ldots$ & . &.. & $\ldots$ & $\ldots$ & $\ldots$ & $\ldots$ &.. & $\ldots$ & $\ldots$ & .. & $\ldots$ & $\ldots$ & $\cdots$ & $\cdots$ & $\ldots$ & $\cdots$ & . & $\cdots$ & $\cdots$ \\
\hline .. & .. & .. &.. & . & .. & . & .. & 1 & $\ldots$ & .. & .. & .. & .. & . & .. & .. & . & .. & . &. & 1 &.. & 1 & . & $\ldots$ & 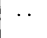 \\
\hline$\cdots$ & .. &.. & $\cdots$ & $\ldots$ & $\ldots$ & $\cdots$ & $\cdots$ & $\cdots$ &.. & $\cdots$ & $\because$ & $\cdots$ & .. & . & . & $\ldots$ & $\ldots$ & $\ldots$ & $\because$ & $\cdots$ & $\ldots$ & $\ldots$ & $\ldots$ & .. & $\cdots$ & 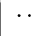 \\
\hline .. & &.. &.. &.. & $\ldots$ &.. & $\ldots$ &.. &.. &. & 1 & $\ldots$ & $\ldots$ & .. & $\because$ & $\ldots$ & . & $\ldots$ & 1 & $\cdots$ & $\therefore$ &. &. & . & 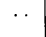 & . \\
\hline$\because$ &.. & .. &.. & . & .. & $\ldots$ & $\ldots$ &.. &.. & . & $\ldots$ & $\ldots$ & $\ldots$ & .. & 1 &.. & $\ldots$ & $\ldots$ & .. & $\ldots$ &.. & $\ldots$ & $\ldots$ & .. & $\ldots$ & .. \\
\hline 6 & .. & .. & 4 & 1 & 3 & 2 & 1 & $\ldots$ & $\ldots$ & 6 & 2 & 1 & 3 & 1 & 71 & 5 & 2 & 1 & 48 & 1 & 1 & 19 & 3 & 5 & 3 & 2 \\
\hline k. &.. & $\ldots$ & . & .. & . & . & $\ldots$ & . &.. & . & $\ldots$ & . & . & $\ldots$ & .. & 2 & . & $\ldots$ & $\ldots$ & . & . & $\ldots$ & . & $\ldots$ & $\ldots$ & . \\
\hline Il & 9 & 3 & 5 & 3 & 2 & 2 & 27 & 1 & $\ldots$ & 7 & . & 4 & 3 & 4 & 6 & 2 & 6 & 3 & 7 & 4 & 4 & 3 & 4 & 9 & $\ldots$ & 2 \\
\hline.. & .. & 1 & .. & .. & . & .. & . & .. & - & $\ldots$ & . & . & $\ldots$ & . &.. & .. & .. & $\ldots$ & .. & .. & $\ldots$ & $\ldots$ & . & .. & $\ldots$ &.. \\
\hline .. & .. &.. &. & $\ldots$ & $\ldots$ & $\ldots$ & $\ldots$ & $\ldots$ & . & $\ldots$ & $\ldots$ & $\ldots$ & $\ldots$ & . & $\ldots$ & .. & .. & $\ldots$ & . & $\ldots$ & $\ldots$ & $\ldots$ & $\ldots$ & $\ldots$ & . & $\ldots$ \\
\hline .. & .. & .. & . & . & $\ldots$ & $\ldots$ & 1 & $\ldots$ & 1 & . & $\ldots$ & $\ldots$ & $\ldots$ & $\ldots$ & $\ldots$ & $\ldots$ & $\ldots$ & $\ldots$ & 1 & $\ldots$ & $\ldots$ & 1 & $\ldots$ & $\ldots$ & 1 & $\ldots$ \\
\hline .. & .. & .. & .. & . & 1 &.. & .. & . & .. & $\ldots$ & . & $\ldots$ & $\ldots$ & $\ldots$ & $\ldots$ & .. & $\ldots$ & $\ldots$ & . & .. & $\ldots$ & .. & $\ldots$ & $\ldots$ & $\ldots$ & $\ldots$ \\
\hline$i$ & .. & $\ldots$ & . & . & $\ldots$ & $\ldots$ & $\ldots$ & $\ldots$ & .. & $\ldots$ & . & $\ldots$ & . & $\ldots$ & .. & $\ldots$ & $\ldots$ & $\ldots$ & $\ldots$ & $\ldots$ & 1 & .. & $\ldots$ & $\ldots$ & $\ldots$ & $\ldots$ \\
\hline$!$ & .. & .. & .. & $\ldots$ & $\ldots$ & $\ldots$ & $\ldots$ & $\ldots$ & $\ldots$ & 1 & $\ldots$ & $\ldots$ & 2 & $\ldots$ & $\ldots$ & $\ldots$ & $\ldots$ & $\ldots$ & $\ldots$ & 1 & . & 1 & 1 & 1 & . & $\ldots$ \\
\hline. & .. & $\ldots$ & .. & .. & $\ldots$ & . & $\ldots$ & . & $\ldots$ & .. & . & $\ldots$ & .. & $\ldots$ & .. & $\ldots$ & $\ldots$ & $\ldots$ & . & $\ldots$ & .. & $\ldots$ & $\ldots$ & $\ldots$ & . & $\ldots$ \\
\hline$\because$ & .. &.. &. & $\ldots$ & . & $\ldots$ & . & $\cdots$ &. & . & $\cdots$ & 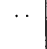 &.. & $\ldots$ & $\ldots$ & .. &.. & . & . & . & $\ldots$ & $\cdots$ & . & . & $\cdots$ & . \\
\hline 1 & .. & . & . & . & 1 & . & . & $\ldots$ & $\ldots$ & $\ldots$ & $\ldots$ & 3 & 4 & $\ldots$ & $\ldots$ & . & . & 2 & $\ldots$ & 1 & $\ldots$ & $\ldots$ & $\ldots$ & $\ldots$ & $\ldots$ & .. \\
\hline 2 & .. & .. & $\ldots$ & $\ldots$ &. & $\ldots$ & $\ldots$ & $\ldots$ & .. & $\ldots$ & $\ldots$ & $\ldots$ & 1 & $\ldots$ & $\ldots$ & . & .. & $\ldots$ & $\ldots$ & 1 & $\ldots$ &.. & $\ldots$ & $\ldots$ &.. & .. \\
\hline .. & .. &.. &.. & .. &.. &. &.. & $\ldots$ &.. & 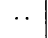 &. & .. & $\ldots$ & .. &. &. & 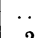 &.. & $\ldots$ & $\cdots$ & . & . & $\cdots$ & . & 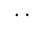 & . \\
\hline .. & .. &.. & 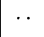 & . & $\therefore$ &.. & $\ldots$ & . & $\cdots$ & 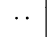 &. & $\cdots$ & $\ldots$ & $\ldots$ & $\ldots$ & . & 2 & $\ldots$ & $\cdots$ & $\cdots$ & $\cdots$ & . & . &.. & 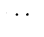 & $\cdots$ \\
\hline$\because$ & .. & .. &.. & . &.. & $\cdots$ & . & . & 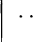 & . & $\cdots$ & $\ldots$ & 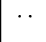 &.. & $\cdots$ &.. & $\ldots$ & . & $\cdots$ & $\ldots$ &.. & $\cdots$ & 1 & . & . & $\cdots$ \\
\hline
\end{tabular}


Alloperla (Assume a Poisson distribution with $\mathrm{n}$ independent trials.)

$\lambda=$ mean number of organisms per sample

$\hat{\lambda}=$ observed mean $=1.54$

$\mathrm{P}$ (the probability that all $\mathrm{n}$ cells will be empty $)=$

$$
\begin{aligned}
& \mathrm{e}^{-\mathrm{n} \hat{\lambda}} \leqq .05 \\
& \mathrm{n} \geqq \frac{\log \epsilon .05}{-\hat{\lambda}}=\frac{-2.99573}{-1.54} \\
&=1.95 \\
& \text { or } \mathrm{n}=2
\end{aligned}
$$

Brachycentrus (Assume a binomial distribution, i.e., $\mathrm{n}$ independent samples each with the same probability $(p)$ of having at least one representative in it.)

$\hat{\mathrm{p}}$ (observed proportion of samples with at least one $)=.58$

$1-\hat{\mathrm{p}}=$ (proportion of samples without Brachycentrus $)=.42$

$\mathrm{P}$ (the probability that all $\mathrm{n}$ cells will be empty) $=$

$$
\begin{aligned}
&(1-\hat{\mathrm{p}})^{\mathrm{n}} \leqq .05 \\
& \mathrm{n} \geqq \frac{\log _{\epsilon} .05}{\log \epsilon_{\epsilon}(1-\hat{\mathrm{p}})}=\frac{-2.99573}{-.86750} \\
&=3.45 \\
& \text { or } \mathrm{n}=4
\end{aligned}
$$

From the figures in table 4 it is clear that 2 or 3 samples would be sufficient to insure (at the 95 per cent level of significance) that at least 1 representative of each of the commonest genera of bottom-dwelling insects would be present. At the ordinal level the required number of samples drops to 1 or 2 . In general the numbers for individual columns fall within the above ranges but some are higher and some lower.

Personal Bias in Sampling. Data from four of the five samplers showed no significant differences. The fifth person showed a consistent bias which amounted to the equivalent of sampling 1.57 times the area covered by the other samplers. Presumably he did not follow directions, but consistently included rocks outside of the square foot outlined by the frame of the Surber sampler. The fact that four persons obtained such parallel results indicates that it is not necessary to have the same man take every sample. On the other hand, the deviation by one sampler clearly demonstrates the necessity for adequate training in sampling techniques. A correction factor was used to reduce both weights and numbers to the corresponding norm obtained by the other four samplers.

Row and Column Effects. Analysis of the data on total numbers and weights revealed no significant row effects. The differences between row means could be attributable to chance variation. Column effects, however, were highly significant. Even when depth and velocity effects were taken out to a first approximation (linear or a grouping) there still remained significant differences between column means. The biological reasons for the 'ross-sectional distribution of various genera presented in figures 4 to 22 are not known. One explanation might be that most aquatic organisms (note Rhithrogena, figure 10, and Glossosoma, figure 14, for striking exceptions) find conditions more to their liking in shallow water, where the velocity of flow is slower and general living conditions less rigorous, than in deeper, faster water. Needham (1928), in discussing the distribution of bottom foods 
in terms of stream width, found a general decrease of 11.9 per cent by weight from shorelines to midchannels of streams over 18 feet wide. Leger (1910) indicated a decrease of 50 per cent in food organisms from the shoreline to midchannels of streams over 5 meters wide. The latter work was done in the streams of southern France.

Correlation Between Types of Organisms, Depth, and Velocity. Striking correlations were found with depth and speed of current. Depth and velocity followed a similar pattern, being lowest in column 1, building up gradually to a peak in columns 7 and 8, and dropping off rapidly to column 10 .

TABLE 4

NUMBER OF SAMPLES REQUIRED TO BE REASONABLY SURE (95 PER CENT) THAT AT LEAST ONE OF A GROUP OF ORGANISMS IS PRESENT

\begin{tabular}{|c|c|}
\hline Organisms & $\begin{array}{l}\text { Number samples } \\
\text { required }\end{array}$ \\
\hline Alloperla $\ldots . .$. & $2-3$ \\
\hline Isogenus......... & $10-15$ \\
\hline 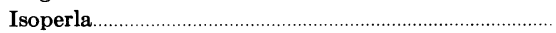 & $12-17$ \\
\hline PLECOPTERA & 2 \\
\hline Baetis & $2-3$ \\
\hline Cinygmula & 2 \\
\hline Rhithrogena.......................... & 2 \\
\hline 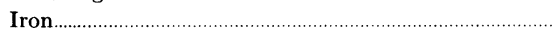 & 2 \\
\hline Ephemerella ................................. & 2 \\
\hline EPHEMEROPTERA & $1-2$ \\
\hline 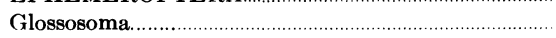 & $2-3$ \\
\hline Hydropsyche & $6-9$ \\
\hline Brachycentrus & $4-5$ \\
\hline Lepidostoma & $3-4$ \\
\hline Rhyacophila & $9-13$ \\
\hline TRICHOPTERA & 1 \\
\hline Simuliidae & 3 \\
\hline Chironomidae & 2 \\
\hline DIPTERA & 1 \\
\hline
\end{tabular}

Rhithrogena (fig. 10) followed this pattern exactly, indicating a definite preference for deep, fast-flowing water. On the other hand, Alloperla (fig. 4), Isoperla (fig. 6), Cinygmula (fig. 9), Ephemerella (fig. 12), Hydropsyche (fig. 15), Rhyacophila (fig. 18), Brachycentrus (fig. 16), and Lepidostoma (fig. 17) showed almost exactly opposite patterns with higher numbers in the shallower, slower water and lower numbers in the deep, swift waters of columns 6 and 7 . The remaining genera did not show consistent trends in their distributional patterns.

Members of the order Trichoptera present a special problem in quantitative sampling of stream beds because some genera are free living whereas others are case-bearers. The question here is how many samples would be required for an investigator to be reasonably sure of getting at least 1 of the casebearing forms as compared with the number required to get at least 1 of the free-living larvae? The figures for the Prosser Creek test are: 2 samples for the case-bearers and 6 to 13 samples for the free-living forms. 


\section{Conclusions}

The Prosser Creek test showed conclusively that an excessive number of samples would be required to provide significant data on total weights and total numbers of bottom organisms. As noted above, the figure for total numbers was 73 , and this was for a single, relatively uniform riffle. Such a conclusion is not surprising, although the figure is higher than most limnologists would have guessed. These results indicate that existing data on stream productivity must be used with caution. However, they also show that only 2 square foot samples are necessary to be reasonably certain of obtaining representatives of the principal groups of organisms present. This is important in connection with stream-pollution surveys, where the total spectrum of groups of organisms is more significant than total weights or numbers (Patrick, 1949).

\section{Summary}

1. Using the Latin Square experimental design, 100 bottom samples of macrofauna were taken in a single riffle in Prosser Creek near Truckee, Nevada County, California, with the Surber Square Foot Sampler, on July 1 and $2,1953$.

2. The samples were preserved in 70 per cent alcohol in the field, after which the organisms were sorted from the trash, and total wet weights of each determined. Sorting and counting were carried to the generic level where possible.

3. Actual wet weights varied from a low of 0.015 gram to a high of 2.31 grams and averaged 0.575 gram of organisms. Numbers varied from 2 to 198 and averaged 75.7 per sample.

4. Statistical analyses of the data revealed that 194 samples would be required to give significant figures (95 per cent level of confidence) as to total wet weight of organisms, and 73 samples would be necessary to give significant figures as to total numbers. The coefficients of variation were 0.78 for weights and 0.56 for numbers.

5. It was found that 2 or 3 samples of the commonest genera of Plecoptera, Ephemeroptera, Trichoptera, and Diptera would be sufficient to insure that at least 1 representative of each would be present.

6. Consistent results were obtained by four of the five persons who took the 100 samples; therefore, with proper training, it is unnecessary to have the same man take every sample.

7. No correlation was found with type of bottom but striking correlations were observed with depth and speed of current.

8. The bulk of genera of aquatic insects indicated a definite preference for shallower, slower water although some, such as the beautifully streamlined Rhithrogena, preferred the deepest, swiftest water. 


\section{Acknowledgments}

The Prosser Creek test was financed by the California State Water Pollution Control Board through the California Department of Fish and Game. Mr. Vinton Bacon of the former agency and Mr. Robert Paul of the latter, were especially helpful in all phases of the program. For aid in taking the test samples in Prosser Creek and in sorting and identifying the organisms, we wish to thank Mr. Elbert Brock, Mr. Albert C. Jones, Mr. Donald Weidlein, and Mr. Lawrence Helm. Mr. Richard Gard assisted in statistical analyses of the data. We are especially indebted to Dr. Evelyn Fix of the Statistical Laboratory of the University of California for her labors on helping design the Prosser Creek test and for her quantitative analysis of the data. Mr. Charles Warren, now Assistant Professor of Fishery Biology at Oregon State College, also gave help in setting up the statistical design for the Prosser Creek test.

EKMAN, S.

\section{LITERATURE CITED}

1911. Neue Apparate zur Qualitativen and Quantitativen Erforschung der Bodenfauna der Seen. Internatl. Rev. Hydrobiol. $7: 164-204$.

Hess, A. D.

1941. New limnological sampling equipment. Limnological Society of America, Special Publication no. 6, 5 pp.

LEGER, L.

1910. Principes de la Methods Rationelle du Peuplement des Cours d'eau a Salmonides. Travaux du Laboratoire de Pisciculture de L'Université de Grenoble, Fascicle 1, p. 531.

NEEDHAM, P. R.

1928. A quantitative study of the fish food supply in selected areas. A Biological Survey of the Oswego River System, N. Y State Cons. Dept. Suppl. to 17th. Ann. Rpt. (1927), pp. 192-206.

PATRICK, RUTH

1949. A proposed biological measure of stream conditions, based on a survey of the Conestoga Basin, Lancaster County, Pennsylvania. Acad. Nat. Sci. Phila. Proc. $100: 283-341$.

Peterson, C. G. J.

1911. Valuation of the sea. I. Danish Biol. Sta. Rpt. I, vol. $20: 1-76$.

SURBER, EugENE W.

1936. Rainbow trout and bottom fauna production in one mile of stream. Amer. Fisheries Soc. Trans. 66:193-202.

Usinger, R. L., and P. R. NEedHaM

1956. A drag-type riffle bottom sampler. The Progressive Fish-Culturist, U. S. Fish and Wildlife Service, Washington, D.C., $18: 42-44$.

Wene, G., and E. L. WiCKLIFF

1940. "Basket" method of bottom sampling. Canadian Ent. $72: 131-35$. 

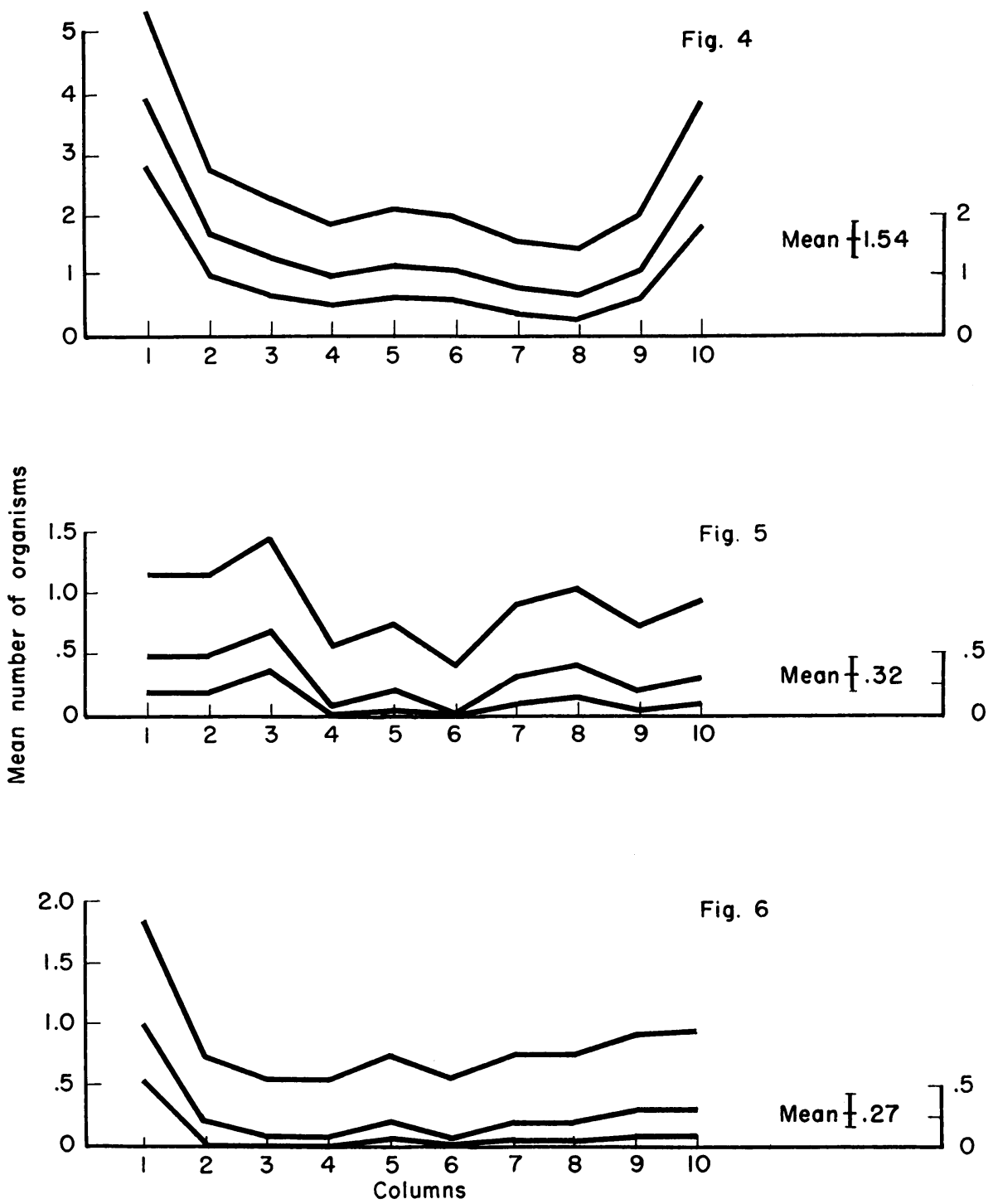

Fig. 4. Alloperla, mean numbers per unit area and upper and lower 95 per cent confidence limits.

Fig. 5. Isogenus, mean numbers per unit area and upper and lower 95 per cent confidence limits.

Fig. 6. Isoperla, mean numbers per unit area and upper and lower 95 per cent confidence limits. 

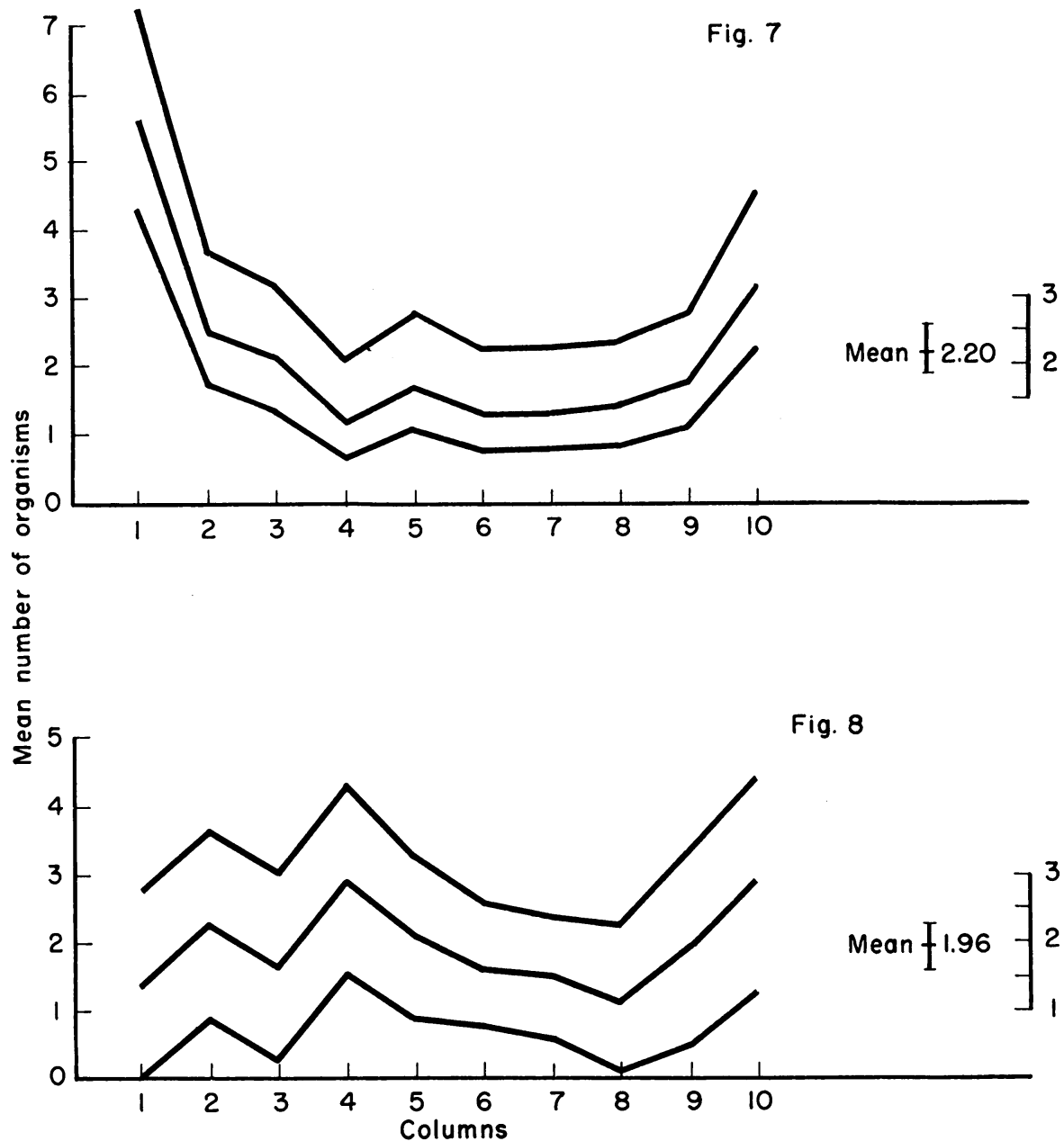

Fig. 7. Plecoptera, mean numbers per unit area and upper and lower 95 per cent confidence limits.

Fig. 8. Battis, mean numbers per unit area and upper and lower 95 per cent confidence limits. 


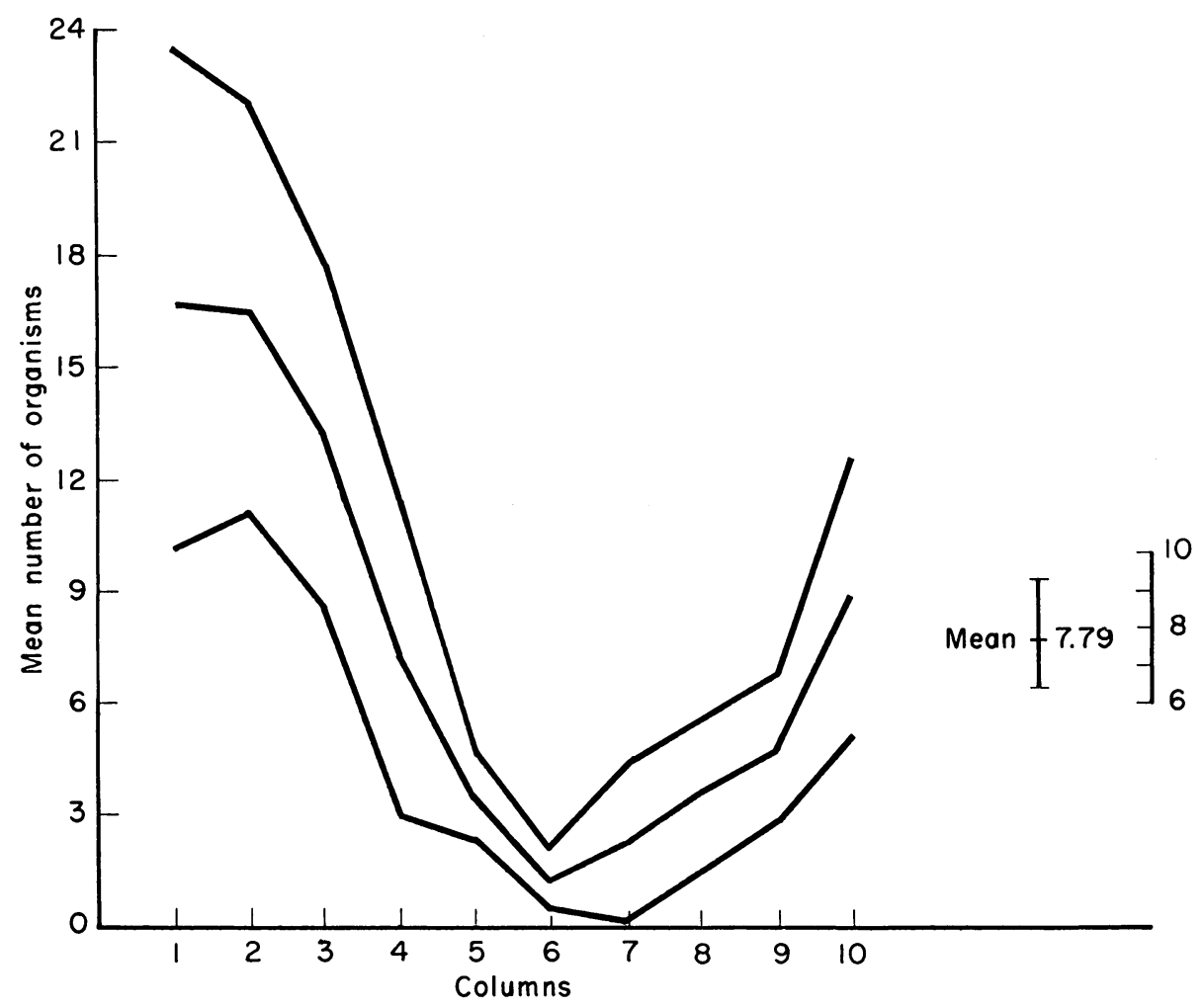

Fig. 9. Cinygmula, mean numbers per unit area and upper and lower 95 per cent confidence limits. 


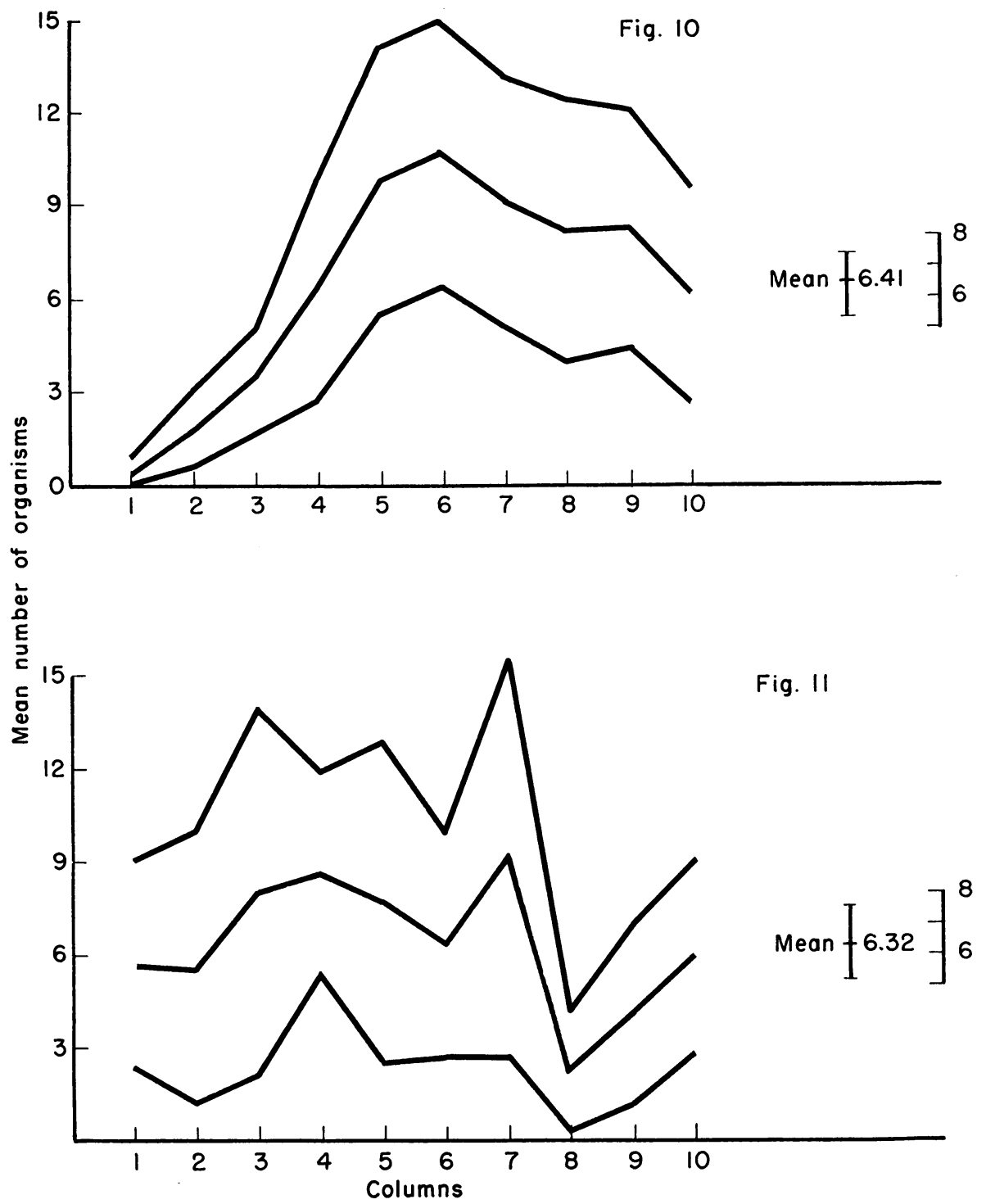

Fig. 10. Rhithrogena, mean numbers per unit area and upper and lower 95 per cent confidence limits.

Fig. 11. Iron, mean numbers per unit area and upper and lower 95 per cent confidence limits. 


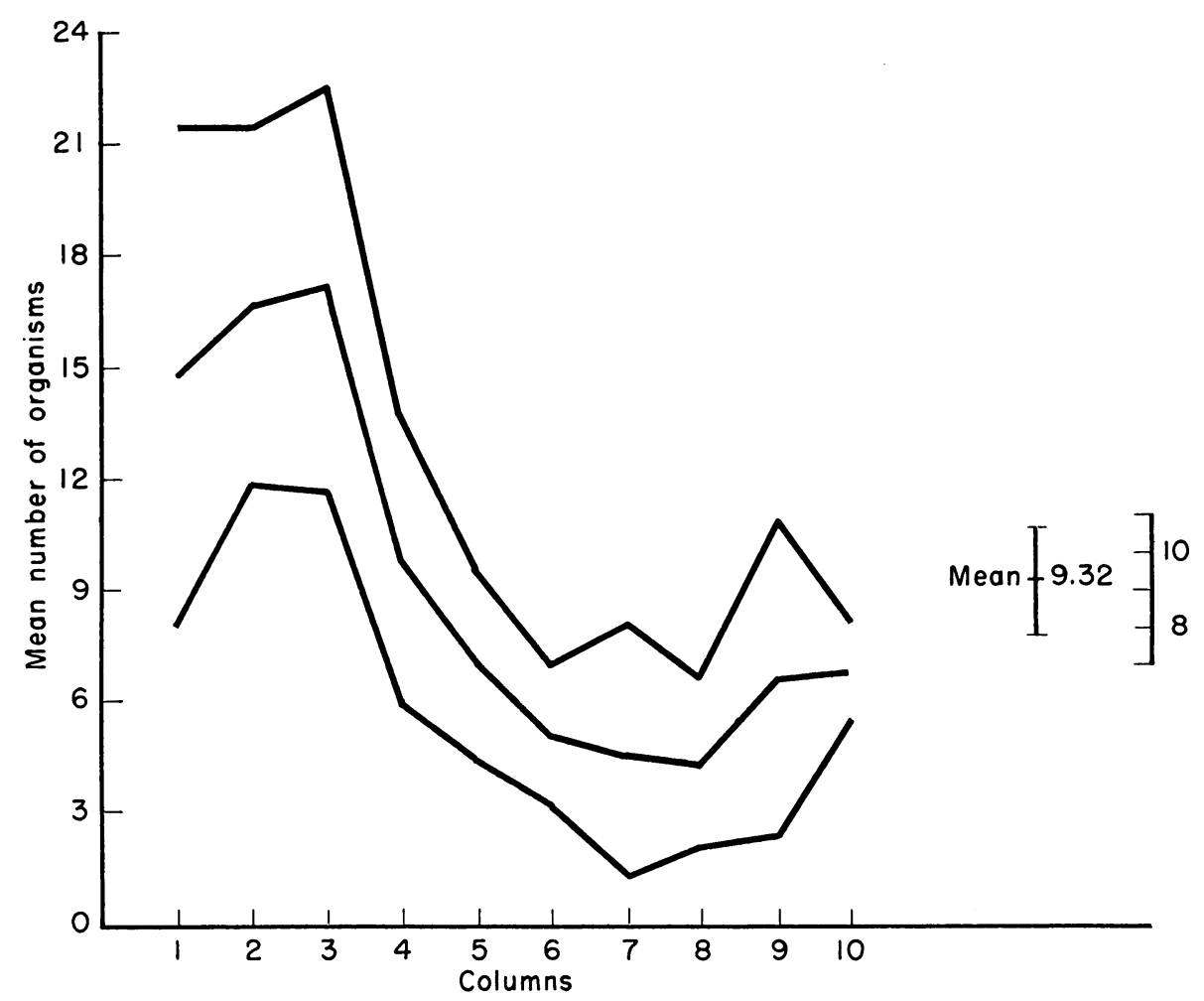

Fig. 12. Ephemerella, mean numbers per unit area and upper and lower 95 per cent confidence limits. 


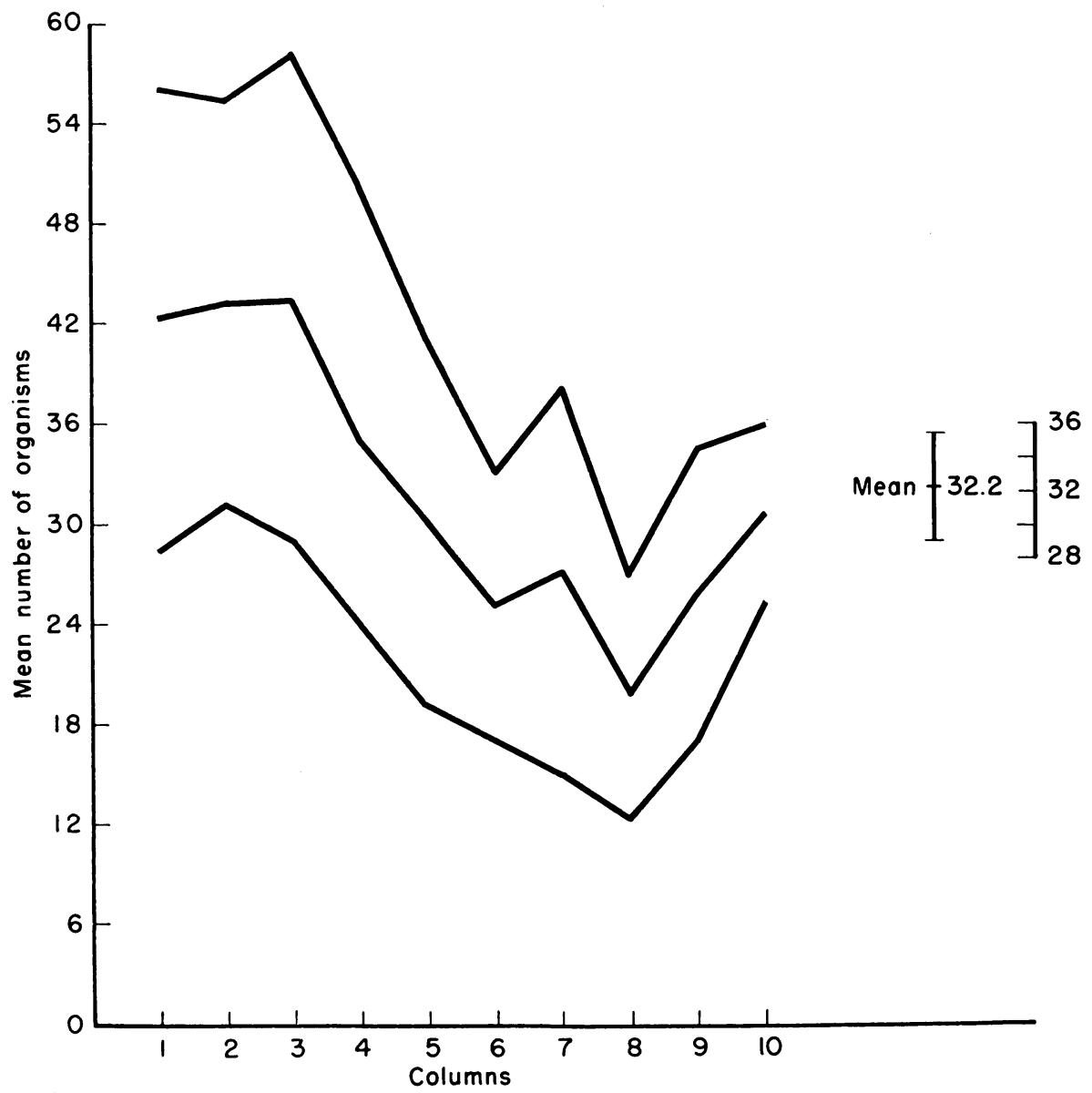

Fig. 13. Ephemeroptera, mean numbers per unit area and upper and lower 95 per cent confidence limits. 


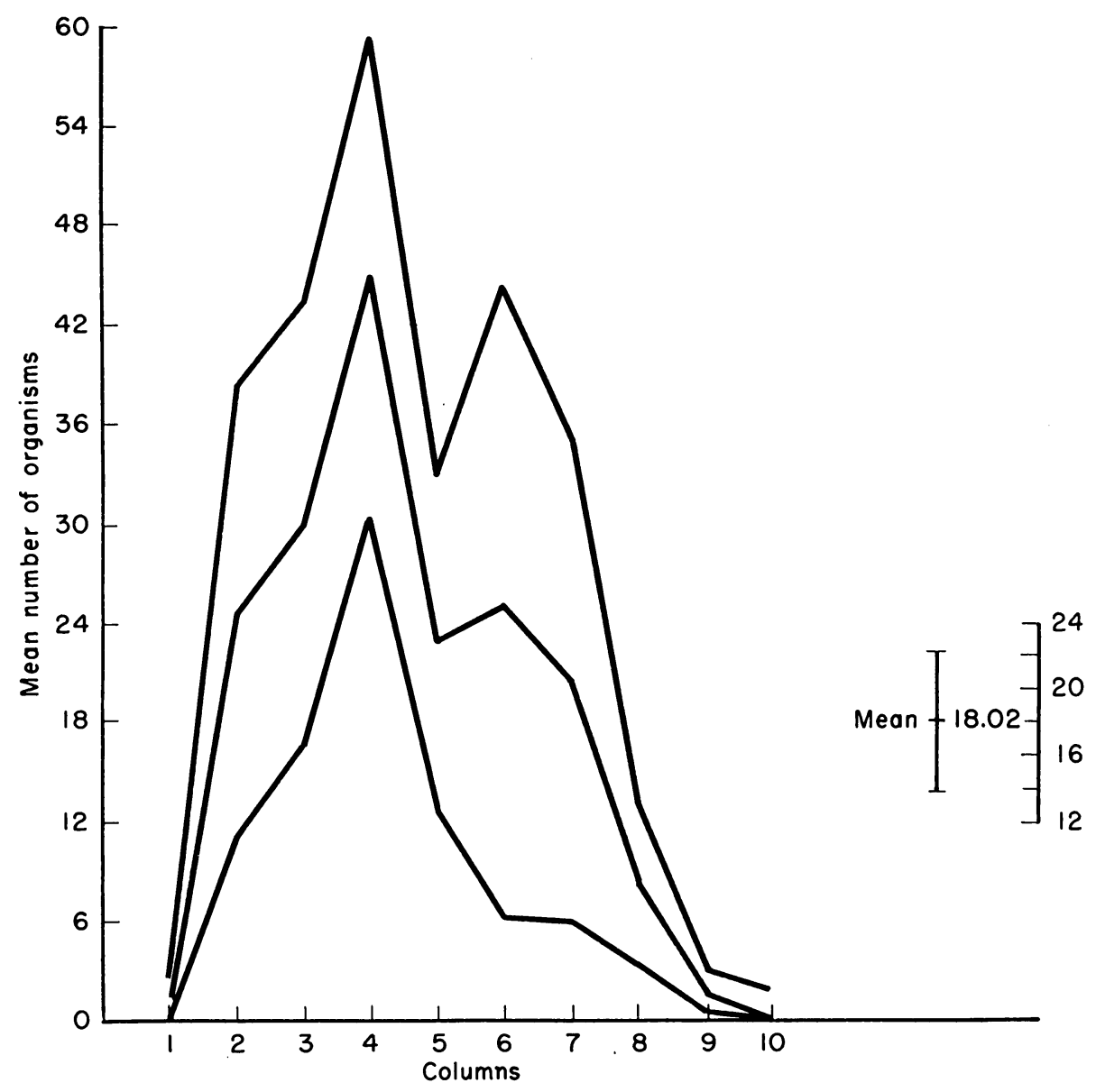

Fig. 14. Glossosoma, mean numbers per unit area and upper and lower 95 per cent confidence limits. 


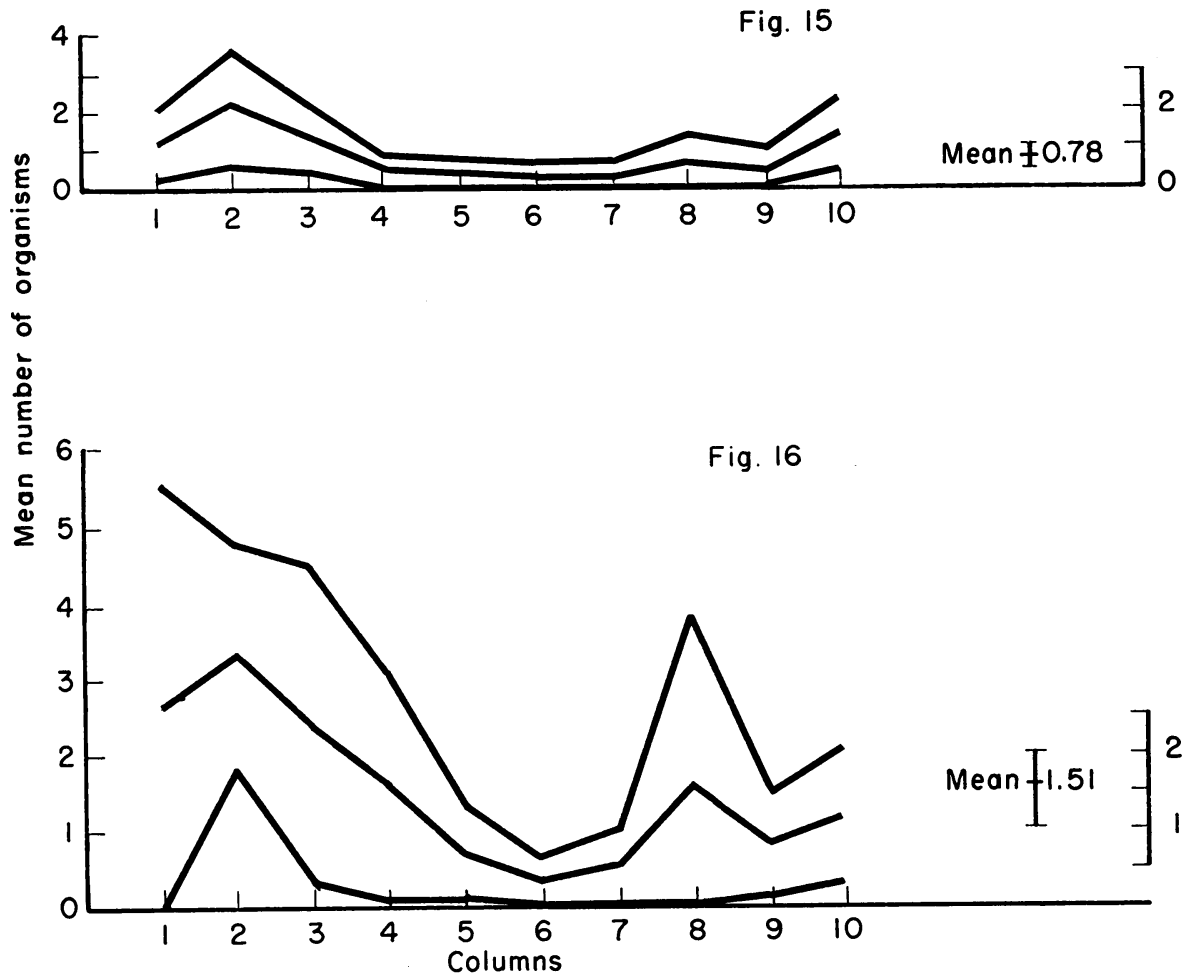

Fig. 15. Hydropsyche, mean numbers per unit area and upper and lower 95 per cent confidence limits.

Fig. 16. Brachycentrus, mean numbers per unit area and upper and lower 95 per cent confidence limits. 


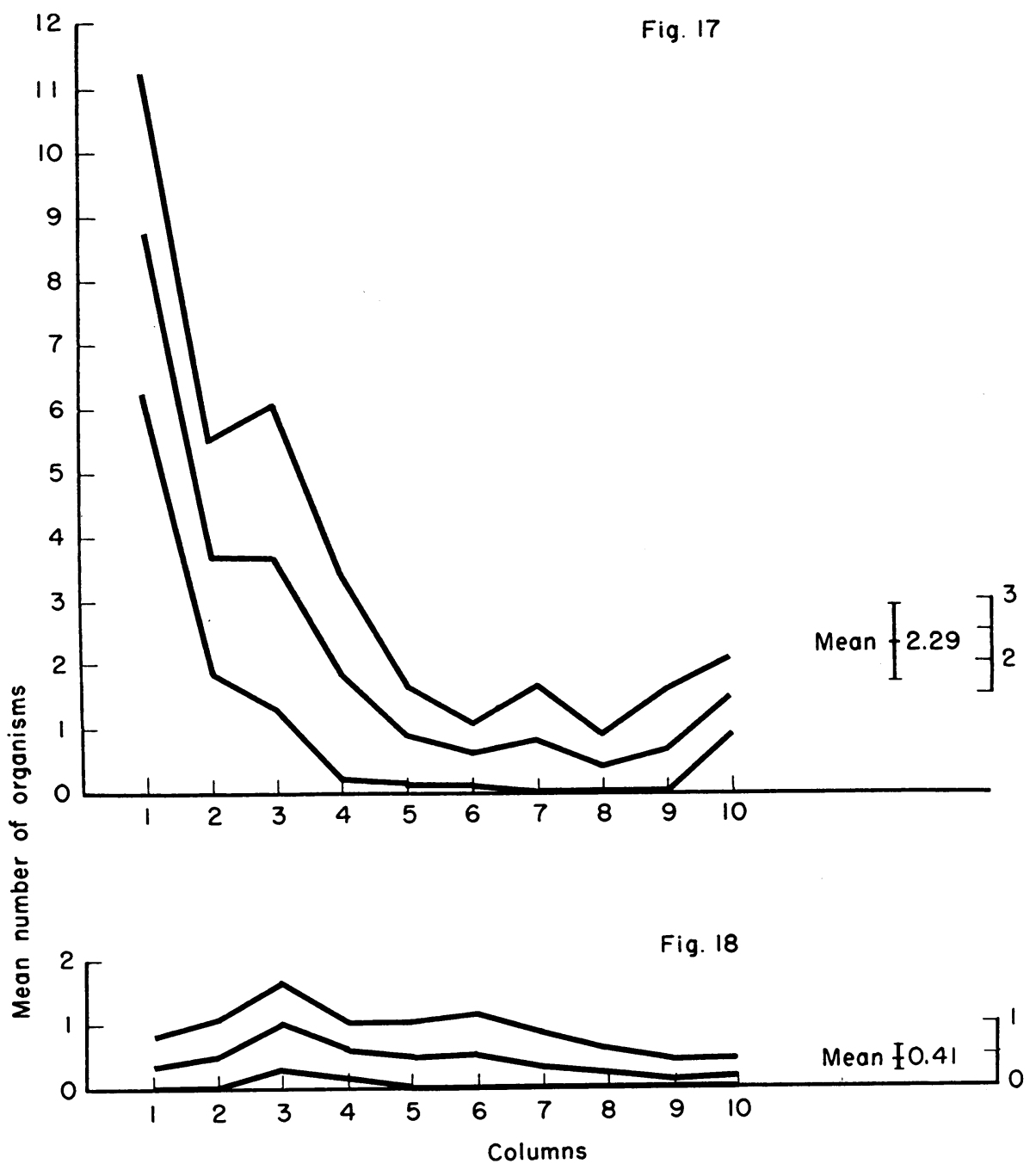

Fig. 17. Lepidostoma, mean numbers per unit area and upper and lower 95 per cent confidence limits.

Fig. 18. Rhyacophila, mean numbers per unit area and upper and lower 95 per cent confidence limits. 


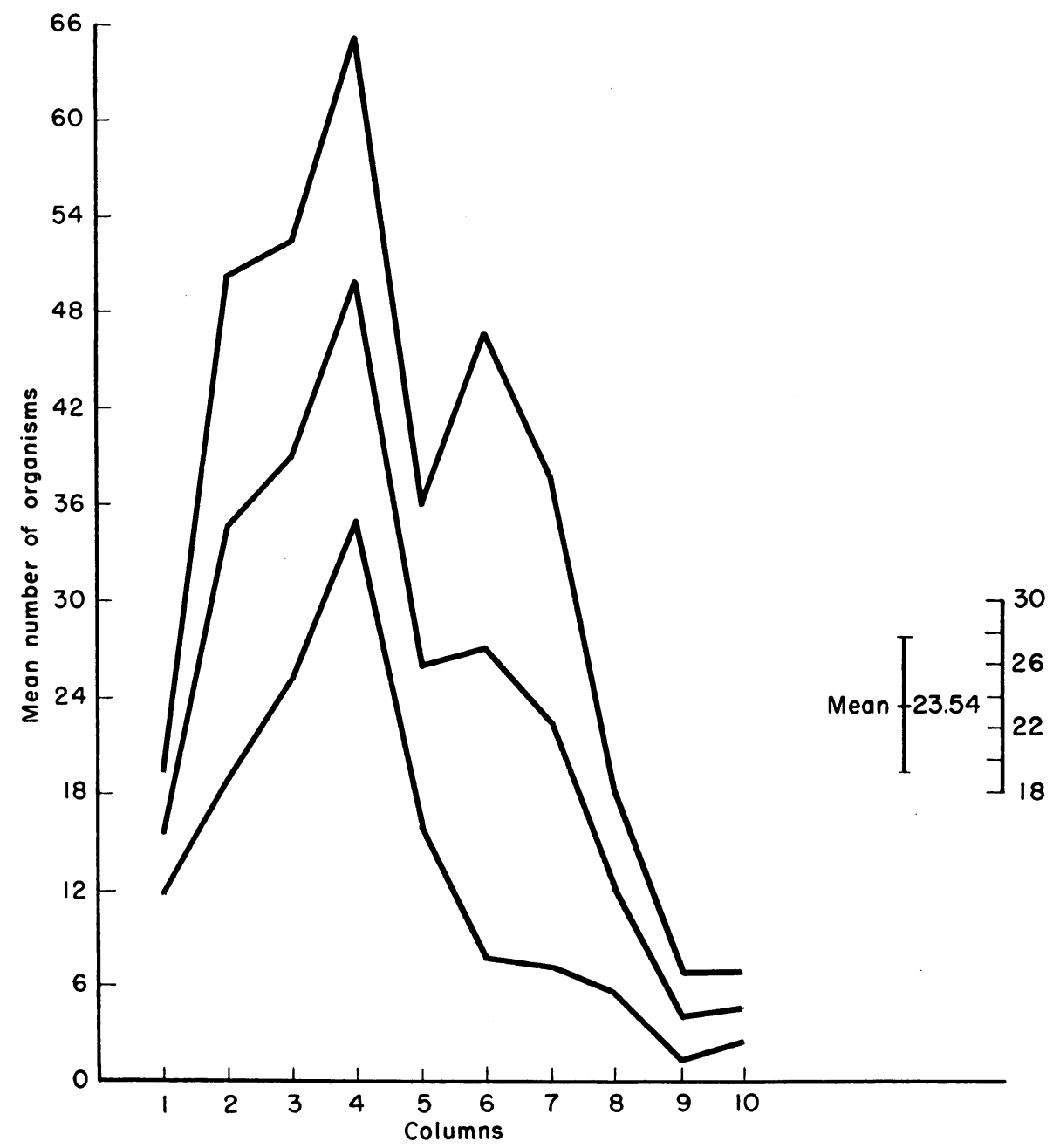

Fig. 19. Trichoptera, mean numbers per unit area and upper and lower 95 per cent confidence limits. 


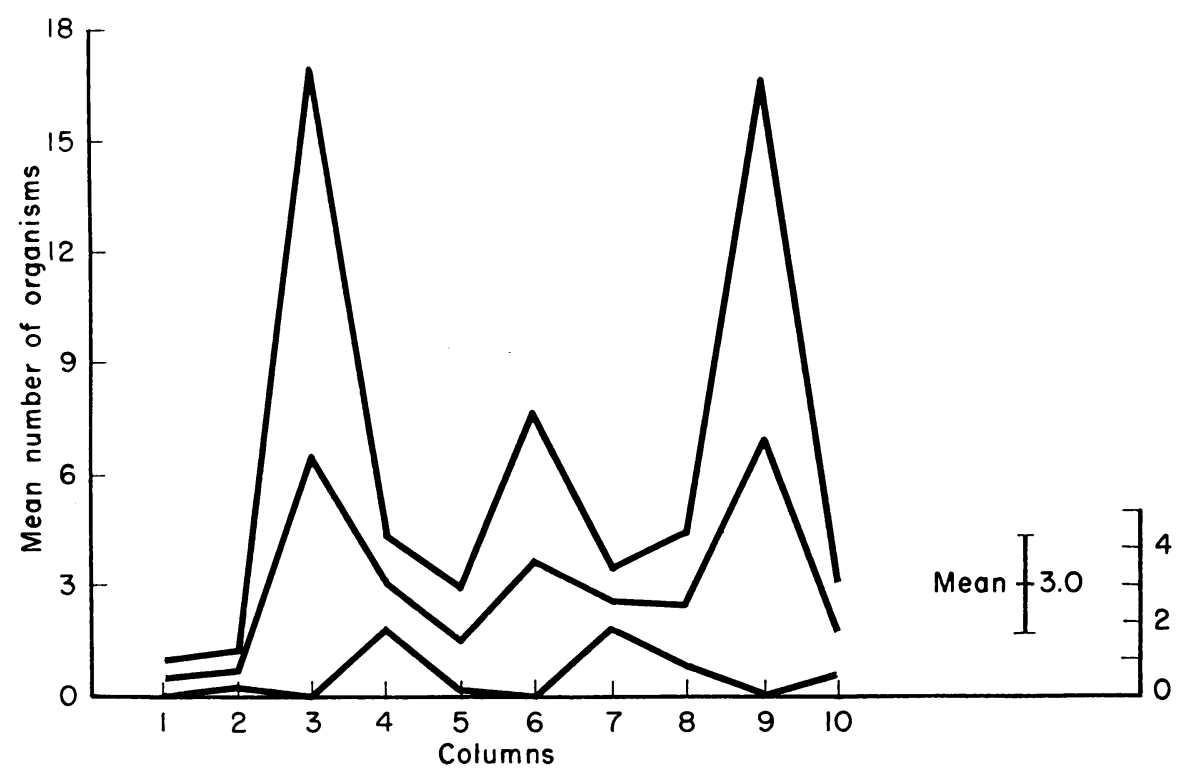

Fig. 20. Simuliidae, mean numbers per unit area and upper and lower 95 per cent confidence limits. 


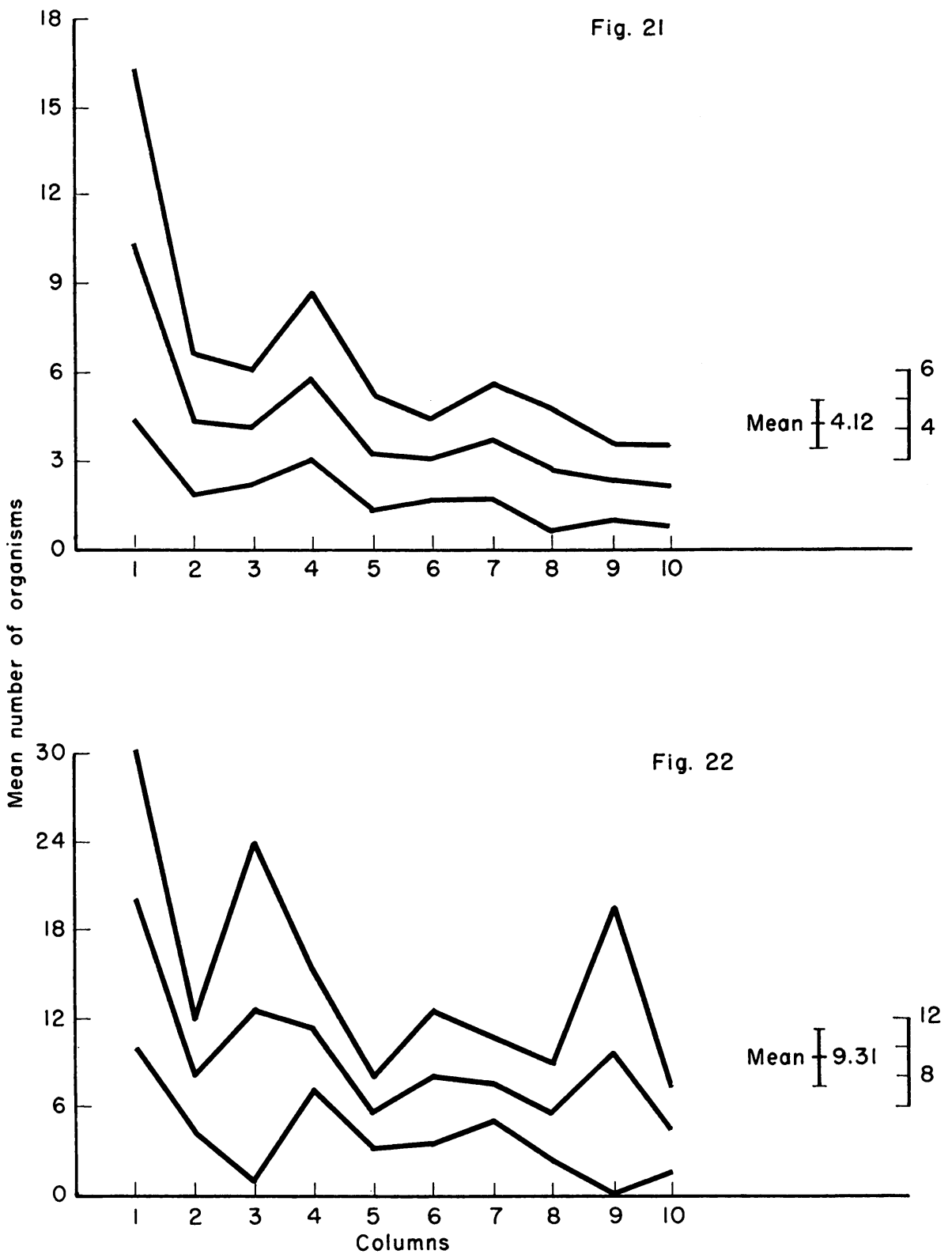

Fig. 21. Chironomidae, mean numbers per unit area and upper and lower 95 per cent confidence limits.

Fig. 22. Diptera, mean numbers per unit area and upper and lower 95 per cent confidence limits. 

The journal Hilgardia is published at irregular intervals, in volumes of about 600 pages. The number of issues per volume varies.

Subscriptions are not sold. The periodical is sent as published only to libraries, or to institutions in foreign countries having publications to offer in exchange.

You may obtain a single copy of any issue free, as long as the supply lasts; please request by volume and issue number from:
Agricultural Publications
Room 22, Giannini Hall
College of Agriculture
University of California
Berkeley 4 , California

The limit to nonresidents of California is 10 separate issues on a single order. A list of the issues still available will be sent on request.

In our publications it is sometimes convenient to use trade names of products or equipment rather than or in addition to scientific identifications. In so doing it is unavoidable in some cases that similar products which are on the market under other trade names may not be cited. No endorsement of named products is intended nor is criticism implied of similar products which are not mentioned. 


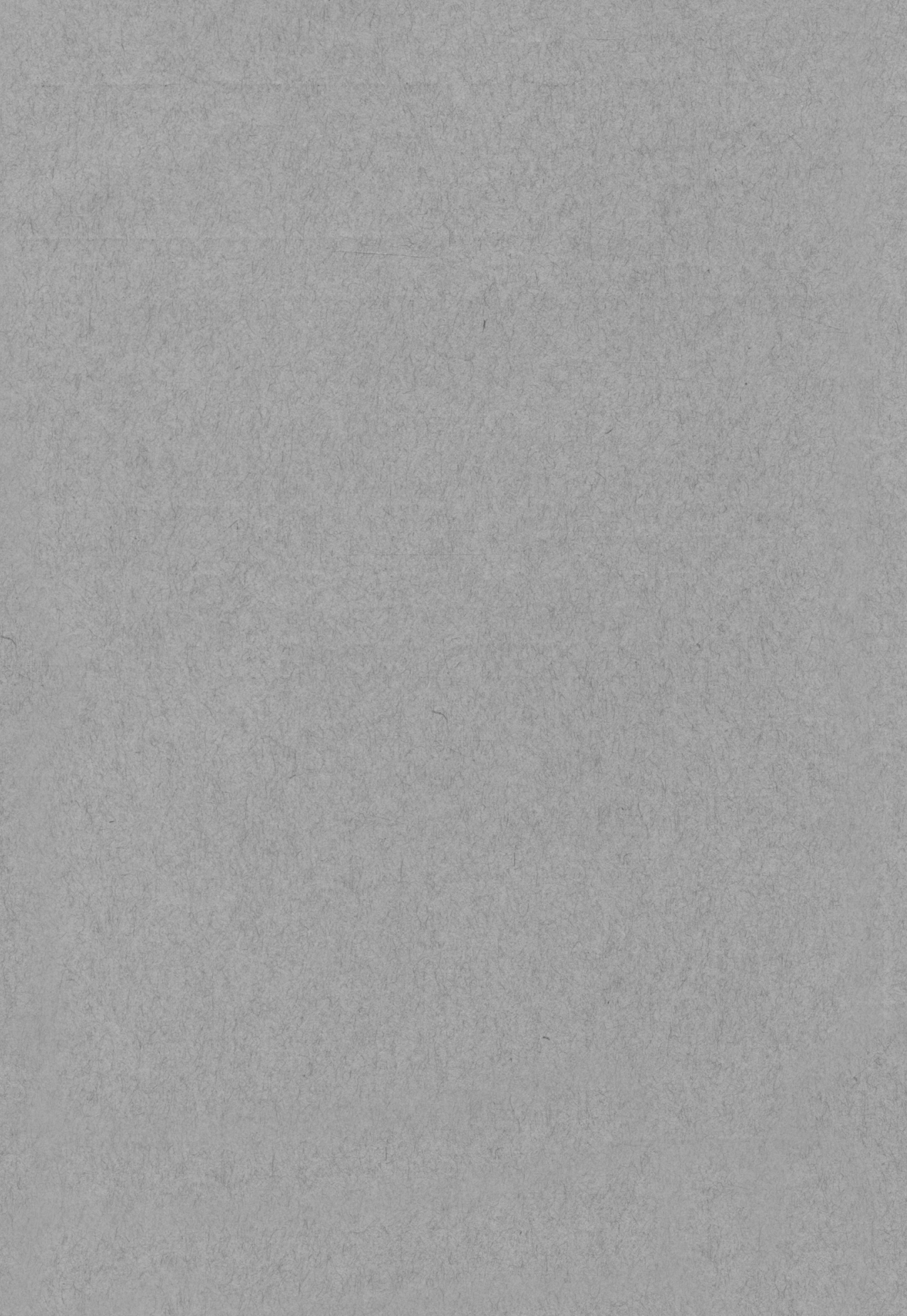

